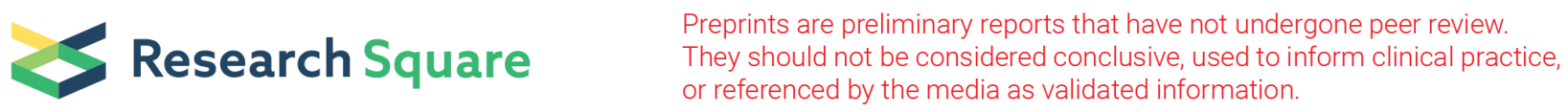

\title{
The Abnormal Metabolites in Tongue Coating of Chronic Gastritis Patients Reflect the Changes in Indexes of Gastroscopic Observation and Gastric Mucosal Pathology
}

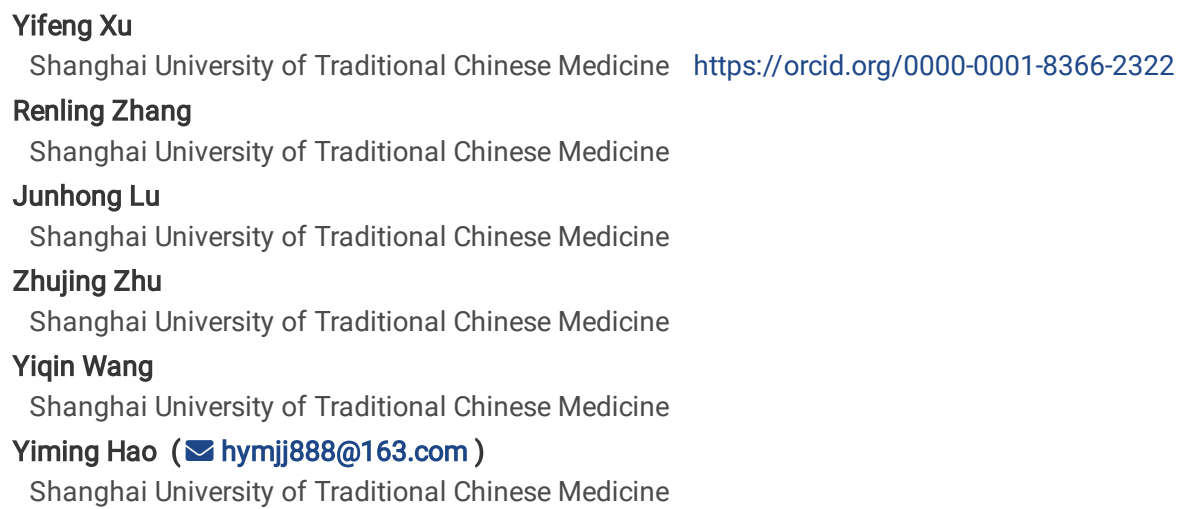




\section{Abstract}

Objective

In this study, we analyzed the correlation between different metabolites in the tongue coating of patients with chronic gastritis, gastroscopy and pathological indexes, and discussed the metabolic mechanism at different pathological stages in the development of chronic gastritis.

\section{Methods}

We used GC-TOF-MS and UHPLC-QE-MS metabonomics to detect the distribution of metabolites in the tongue coating of patients with chronic gastritis, and analyzed the correlation between different metabolites in the tongue coating of patients with chronic gastritis and gastroscopy and pathological indexes.

Results

Compared with 50 healthy people, 54 metabolites were upregulated and 47 metabolites were downregulated in 350 patients with chronic gastritis. The main differential metabolites were Lipids and lipid-like molecules, which contain 47 metabolites. The best diagnostic model was composed of 5 metabolites, with an accuracy of $95.4 \%$, a specificity of $87.4 \%$ and a sensitivity of $88.0 \%$. These 5 metabolites were 1-methyladenosine, Sphinganine 1-phosphate, 3Hydroxycapric acid, 4-Ipomeanol, and Nervonic acid. Compared with healthy people, Sphinganine 1-phosphate, 4-Ipomeanol, and Nervonic acid were significantly upregulated in chronic gastritis patients, and 1-methyladenosine and 3-Hydroxycapric acid were significantly downregulated in chronic gastritis patients. After correlation analysis between differential metabolites in tongue coatings and gastroscopic indexes, we found that Trimethylaminoacetone, Sphinganine1-phosphate, alpha-Carboxy-delta-decalactone, and 5,6-Dihydroxyindole were positively correlated with intestinal metaplasia. Conduritol-betaexpoxide, Tetracosanoic acid, Lactosylceramide(d18:1/26:0), Chondrillasterol 3-[glucosyl-(1->4)-glucoside], Azelaic acid, and 1-Methyladenosine were negatively correlated with intestinal metaplasia. Sphinganine1-phosphate, alpha-Carboxy-delta-decalactone, and 5,6-Dihydroxyindole were positively correlated with atrophic. Octadecanol, conduritol-beta-expoxide, Tetracosanoic acid, Smilanippin A, Lactosylceramide(d18:1/26:0), Chondrillasterol 3-[glucosyl(1->4)-glucoside], and Azelaic acid were negatively correlated with atrophic. 6-deoxyglucitol was negatively correlated with bile reflux, methylmaleic acid, 4methylcatechol, and 2,4-dichloro-1-(2-chloroethenyl)-benzene were negatively correlated with Hp, 3-benzoyloxy-11-oxo-12-ursen-28-oic acid was negatively correlated with gastric mucosal erosion. From the change trend of different metabolites in different pathological stages, we found that the content of conduritol-beta-expoxide decreased significantly in mild atrophic compared with moderate atrophic and the content of conduritol-beta-expoxide decreased significantly in mild intestinal metaplasia compared with moderate intestinal metaplasia.

Conclusions

We found that Lipids and lipid-like molecules were the main abnormal metabolites in patients with chronic gastritis. Among them, Sphinganine 1-phosphate, which was significantly positively correlated with the aggravation of atrophic and intestinal metaplasia, could be used as one of the diagnostic model markers for chronic gastritis. Additionally, the amount of conduritol-beta-expoxide significantly decreased with the aggravation of atrophic and intestinal metaplasia. We believe that these differential markers in tongue coating may help us to establish a noninvasive and convenient auxiliary detection method for gastritis and gastric precancerous lesion in the future.

\section{Introduction}

Nowadays, people are living at an increasing pace, the work pressure is increasing, and the diet structure is changing, more and more people are suffering from digestive system diseases. Studies have shown that chronic gastritis, as one of the most common severe epidemic infections worldwide, has now affected more than half of the people in the world [1]. Chronic gastritis can be divided into chronic atrophic gastritis and chronic non-atrophic gastritis, and it is worth noting that gastric mucosal atrophic may relate to the development of gastric cancer [1]. Therefore, early detection and treatment of chronic gastritis play an important role both in preventing further injury to the disease and improving the quality of life. At present, gastroscopy is the main method of diagnosis and curative effect evaluation of chronic gastritis, which is of great significance for the early detection and treatment of chronic gastritis. However, gastroscopy cannot be performed frequently in a short time [2]. Thus, finding a noninvasive and repeatable monitoring method to observe the progress of chronic gastritis is one of the main directions of our research.

Tongue diagnosis is one of the important diagnostic methods of Traditional Chinese Medicine (TCM), which is non-invasive and repeatable. The main way of tongue diagnosis is to observe the changes of tongue body and tongue coating. According to the theory of TCM, tongue coating is a very sensitive index that can reflect the physiological and pathological state of the spleen and stomach.

In recent years, metabolomics has been widely used in medical research and also used to find diagnostic biomarkers of various diseases. In the study of tongue coating metabolites of chronic gastritis, some researchers used ultra-performance liquid chromatography and mass spectrometry (UPLC-MS) technology or liquid chromatography and mass spectrometry (LC-MS) technology to detect and analyze the substances in tongue coating of chronic gastritis patients. They found that there were differences in tongue coating metabolites between healthy people and chronic gastritis patients [3-4]. However, these studies only used LC-MS techniques, and the sample size was less than 100 cases, used only one detection method and under conditions of low sample size, the metabolites in tongue coating could not be fully reflected. Meanwhile, studies on the correlation of tongue coating metabolites and gastroscopic indexes in chronic gastritis patients have not been reported.

Gas chromatography-time-of-flight-mass spectroscopy (GC-TOF-MS) is also a metabonomic method, GC-TOF-MS has a higher separation ability than liquid chromatography-Q exactive orbitrap-mass spectroscopy (UHPLC-QE-MS) in the analysis of polar metabolites. Besides, the GC-TOF-MS system's mass spectra are more repeatable than UHPLC-QE-MS, which makes GC-TOF-MS libraries more comprehensive. Insufficient is that GC-TOF-MS can only detect more than a 
hundred metabolites while UHPLC-QE-MS can detect thousands of metabolites that contain semi-polar metabolites [5]. Due to the complex composition of metabolites on tongue coating, the combination of two detection methods can be more comprehensively used to detect the characteristics of tongue coating metabolites.

Therefore, this study combined UHPC-QE-MS and GC-TOF-MS technology to detect and analyze the metabolites in 350 patients with chronic gastritis. The abnormal fluctuation of these metabolites is helpful for us to better understand the changes of the metabolites of tongue coating in patients with chronic gastritis from different aspects.

\section{Materials And Methods}

\subsection{Samples}

To explore the composition of tongue coating related metabolites in patients with chronic gastritis, we selected a case-control study approach. We selected 350 chronic gastritis patients in Longhua Hospital (an affiliate of the Shanghai University of traditional Chinese medicine) to perform gastroscopy and pathological examination of gastric mucosa, and selected 50 teachers and students of Shanghai University of traditional Chinese medicine as the healthy control group. People in the selected healthy control group had no history of stomach disease or stomach discomfort at present. The results of their blood cell analysis, liver function, renal function, blood lipid, blood glucose, blood pressure, tumor index, color Doppler ultrasound of neck and abdomen, chest CT and barium metal fluoroscopy were normal. All participants were Chinese (self-reported), they participated in the study from December 2018 to October 2019. Participants underwent gastroscopy and gastric mucosal pathology immediately after being sampled for tongue coating specimens. A summary of their demographic and clinical information was provided in Table 1.

Table 1

Summary of demographics and clinical information of the participants

\begin{tabular}{|c|c|c|}
\hline Demographics and clinical information & Chronic gastritis patient group & Normal control group \\
\hline Sample number & 350 & 50 \\
\hline Ratio of male to female & $1: 0.74$ & 1:0.92 \\
\hline Average age (year) & $45.71 \pm 13.83$ & $34.04 \pm 10.34$ \\
\hline Number (percentage) of samples diagnosed this time & $3(0.86 \%)$ & N/A \\
\hline Number (percentage) of samples diagnosed for less than 10 years & $259(74.00 \%)$ & N/A \\
\hline Number (percentage) of samples diagnosed for $10-20$ years & $71(20.29 \%)$ & $\mathrm{N} / \mathrm{A}$ \\
\hline Number (percentage) of samples diagnosed for $20-30$ years & $15(4.29 \%)$ & N/A \\
\hline Number (percentage) of samples diagnosed for $30-40$ years & $4(1.14 \%)$ & N/A \\
\hline Number (percentage) of samples diagnosed over40 years & $1(0.29 \%)$ & N/A \\
\hline Number (percentage) of samples untreated & $83(23.71 \%)$ & N/A \\
\hline Number (percentage) of samples only taking western medicine & $174(49.71 \%)$ & N/A \\
\hline Number (percentage) of samples only taking traditional Chinese medicine & $180(51.43 \%)$ & N/A \\
\hline Number (percentage) of samples taking western medicine and traditional Chinese medicine & $84(24.00 \%)$ & N/A \\
\hline
\end{tabular}

\subsection{Ethics Approval}

The study was approved by the Ethics Committee of Shanghai University of TCM in China in December 2018 and performed in accordance with the Declaration of Helsinki. All the subjects had signed informed consent agreements before collecting samples.

\subsection{Criteria}

Diagnostic Criteria

1) Endoscopy was performed on patients and biopsies were taken from suspicious lesion sites such as gastric antrum, angle, body and cardia.

2) The six gastroscopic and pathological indexes were inflammatory activity, atrophic, intestinal metaplasia, Helicobacter pylori (Hp), bile reflux and gastric mucosal erosion. The inflammatory activity, atrophic and intestinal metaplasia were divided into four grades: none, mild, moderate and severe.

3) Patients with $\mathrm{Hp}$ infection should confirm that the $\mathrm{Hp}$ test of gastric mucosa was consistent with the positive result of the C13 breath test.

4) The histopathological assessment was performed by two experienced pathologists in accordance with the clinical guidelines of "the updated Sydney System" [6].

Exclusion Criteria 
Patients who had gastroesophageal reflux disease, gastric ulcer, gastric hemorrhage, gastric cancer, duodenal ulcer, or other intestinal diseases were identified by endoscopic examination.

Patients who had diseases of the motor system, nervous system, endocrine system, circulatory system, respiratory system, urinary system, and reproductive system.

Patients who had a mental illness or female subjects were during their pregnancy and lactation period.

Patients who had lesions of the tongue, mouth, nose, or pharynx within one month prior to the collection of samples.

Patients who had received probiotics or antibiotics within one month prior to sample collection.

Patients who had used tobacco or consumed alcohol. The participants had a body mass index (BMI) over 28 [7].

\subsection{Tongue coating samples collection}

Samples of tongue coating were collected before participants had breakfast. Before collection, the participants rinsed the mouth with a stroke-physiological saline solution 3 times in order to remove the residues in the mouth. The collector used a sterile specimen collection swab (CY-98000, iClean, Huachenyang Technology Co., Ltd, CN) to scrape the thick part of the tongue coating of participants 5 times, then put the head of the tongue coating sample swabs into a sterile centrifuge tube. All tongue coating samples were collected by the same researcher. The tongue coating samples were stored for use at $-80^{\circ} \mathrm{C}$.

\subsection{GC-TOF-MS Metabolomics Processing}

The reagents used in the GC-TOF-MS experiment were listed in Supplementary Table S1a. The experimental steps were as follows:

1) Transfer the swab head of the tongue coating sample to a $5 \mathrm{ml}$ sterile Eppendorf (EP) tube for weighing, and add the pre-cold extraction mixture (Vmethanol:Vchloroform = 3:1) $1500 \mu \mathrm{L}$ with internal standard solution (adonitol, $0.5 \mathrm{mg} / \mathrm{mL}$ stock) $15 \mu \mathrm{L}$. The sample underwent ultrasonication in the ice water for $30 \mathrm{~min}$. And then removed the swab head. After centrifugation at $4^{\circ} \mathrm{C}, 10000 \mathrm{rpm}$ for 15 minutes, $500 \mu \mathrm{L}$ supernatant was transferred to fresh tubes.

2) We took $100 \mu \mathrm{l}$ from each sample and combined it together to prepare a quality control (QC) sample. After evaporation in a vacuum concentrator, add $40 \mu \mathrm{L}$ methoxyaminated hydrochloride $\left(20 \mathrm{mg} / \mathrm{ml}\right.$ pyridine), incubated at $80^{\circ} \mathrm{C}$ for $30 \mathrm{~min}$, and then incubated with $60 \mathrm{ml}$ at $70^{\circ} \mathrm{C} \mu \mathrm{L}$ bis-(trimethylsilyl)trifluoroacetamide regent (containing $1 \%$ trimethylchlorosilane, $\mathrm{V} / \mathrm{V}$ ) was derived for $1.5 \mathrm{~h}$. After gradually cooled the sample to room temperature, $5 \mu \mathrm{L}$ fatty acid methyl ester (in chloroform) was added to QC samples.

3) GC-TOF-MS detection was performed using an Agilent 7890 gas chromatograph and TOF mass spectrometer. The system employed a db-5ms capillary column. Inject a sample of $1 \mu \mathrm{l}$ aliquot in a splitless mode. With Helium as the carrier gas, the purge flow at the front inlet was $3 \mathrm{~mL} / \mathrm{min}$, and the gas flow rate through the chromatographic column was $1 \mathrm{~mL} / \mathrm{min}$. The initial temperature was maintained at $50^{\circ} \mathrm{C}$ for $1 \mathrm{~min}$, and then raised to $310^{\circ} \mathrm{C}$ at a rate of $20^{\circ} \mathrm{C} / \mathrm{min}$, then maintained at $310^{\circ} \mathrm{C}$ for $6 \mathrm{~min}$. The implantation, the transfer line, and ion source temperatures were 280,280 , and $250^{\circ} \mathrm{C}$. The energy was $-70 \mathrm{eV}$ in electron collision mode. The mass spectrum data were obtained in full-scan mode with the $\mathrm{m} / \mathrm{z}$ range of $50-500$ at a speed of 12.5 spectrum/s and solvent delay of $6.30 \mathrm{~min}$

4) The original data were analyzed by using Chroma TOF (V 4.3x, LECO) software [8], including peak extraction, baseline adjustment, deconvolution, alignment and integration. The mass spectrum and retention index identification of metabolites were performed by using the LECO-Fiehn Rtx 5 database.

5) The peaks detected in QC samples were less than half of the peaks were removed in QC samples with RSD $>30 \%$ [9].

\subsection{UHPLC-QE-MS Metabolomics Processing}

The reagents used in the UHPLC-QE-MS experiment were listed in Supplementary Table S1. The experimental steps were as follows:

1)Add $1500 \mu \mathrm{L}$ of extract containing the isotopically-labeled internal standard mixture (Vacetonitrile:Vmethanol: Vwater = 2:2:1). After being vortexed for 30 s, the solution was sonicated on ice for $30 \mathrm{~min}$.

2) $-40^{\circ} \mathrm{C}$ for $1 \mathrm{~h}$ and centrifuged at $12000 \mathrm{rpm}$ for 15 minutes at $4^{\circ} \mathrm{C}$. The resulting supernatant was transferred to a fresh glass vial for analysis. The supernatants of all the samples were mixed well to make QC samples.

3) LC-MS detection was performed on a UHPLC system (Vanquish, Thermo Fisher Scientific) with a UPLC BEH Amide column (2.1 mm×100 mm, $1.7 \mu \mathrm{m})$ coupled to Q Exactive HFX mass spectrometer (Orbitrap MS, Thermo). The mobile phases were $25 \mathrm{mmol} / \mathrm{L}$ ammonium acetate and $25 \mathrm{mmol} / \mathrm{L}$ ammonia hydroxide in water $(\mathrm{pH}=9.75)(\mathrm{A})$ and acetonitrile $(\mathrm{B})$. The elution gradient as follows: $0-0.5 \mathrm{~min}, 95 \% \mathrm{~B} ; 0.5-7.0 \mathrm{~min}, 95 \%-65 \% \mathrm{~B} ; 7.0-8.0 \mathrm{~min}, 65 \%-40 \% \mathrm{~B} ; 8.0$ $9.0 \mathrm{~min}, 40 \% \mathrm{~B} ; 9.0-9.1 \mathrm{~min}, 40 \%-95 \% \mathrm{~B} ; 9.1-12.0 \mathrm{~min}, 95 \% \mathrm{~B}$. The column temperature was $25^{\circ} \mathrm{C}$. The auto-injection temperature was $4{ }^{\circ} \mathrm{C}$, and the injection volume was $3 \mu \mathrm{L}$.

4) The QE HFX mass spectrometer acquired MS/MS spectra in the information-dependent acquisition mode under the control of the acquisition software (Xcalibur, Thermo). In this mode, the acquisition software continuously assessed the full scan mass spectrum. The ESI source conditions were set as following: sheath gas flow 50 Arb, auxiliary gas flow 10 Arb, capillary temperature $320^{\circ} \mathrm{C}$, full MS resolution as 60000 , MS/MS resolution as 7500 , collision energy 10/30/60 in nice mode, spray Voltage $3.5 \mathrm{kV}$ (positive) or -3.2 kV (negative), respectively. 
5) The raw data were converted to the mzXML format using ProteoWizard and peak detection, extraction, alignment, and integration were performed using an in-house XCMS based program developed in R.

6) metabolite annotation was adopted from the company's own MS2 database (BiotreeDB V2.1). The cutoff value for annotation was set to 0.3 [10].

\subsection{Statistical analysis}

The peak number, sample names, and standardized peak areas were input into Simca-p+13.0 software (Umea, Sweden) for principal components analysis (PCA) and orthogonal projections to latent structures-discriminant analysis (OPLS-DA). To further validate the model, a drive substitution experiment was carried out. The false discovery rate (FDR) of the rank-sum test and the p-value $(P<0.05)$, variable importance in the projection (VIP) of the first principal component of the OPLS-DA model (VIP>1), similarity value (SV) detected by GC-TOF-MS (SV>700) [11], and MS2 score detected by UHPLC-QE-MS detection (MS2 score>0.6)[12] were used to determine the significant difference of metabolites between the two groups. The mean value of the metabolites peak areas was compared between the two groups, and the log fold change (FC) value was calculated.

Spearman algorithm was used for correlation analysis, and correlation coefficient (Corr) matrix and correlation P-value matrix were calculated for subsequent analysis and graphics drawn.

Correlation analysis was performed using the Spearman algorithm, and the Corr matrix, correlation P-values were calculated for subsequent analysis and graphically plotted.

\section{Results}

\subsection{Distribution of patients with chronic gastritis in different indexes of gastroscopic observation and gastric mucosal pathology}

Table 2 Distribution of patients with chronic gastritis in different indexes of gastroscopic observation and gastric mucosal pathology

\begin{tabular}{lllll} 
indexes of gastroscopic observation and gastric mucosal pathology & Mild & Moderate & Severe & Proportion of total samples, \% \\
\hline inflammatory activity & 48 & 45 & 3 & $96(27.43 \%)$ \\
\hline atrophic & 101 & 22 & 6 & $129(36.86 \%)$ \\
\hline intestinal metaplasia & 87 & 21 & 6 & $114(32.57 \%)$ \\
\hline Hp & 10 & 20 & 19 & $49(14.00 \%)$ \\
\hline bile reflux & N/A & N/A & N/A & $60(17.14 \%)$ \\
\hline gastric mucosal erosion & N/A & N/A & N/A & $171(48.86 \%)$
\end{tabular}

\subsection{Different peaks findings in tongue coating samples}

We performed GC-TOF-MS and UHPLC-QE-MS metabolomic profiling of tongue coating samples from 350 patients with chronic gastritis and 50 healthy controls.

The GC-TOF-MS and UHPLC-QE-MS spectra examples of the same person were shown in Figure 1. As shown in Figure 1a Figure1b and Figure 1c, there were some different mass spectrum peaks between the chronic gastritis patients and healthy control people.

We adopted the criteria of P-value $<0.05$ and VIP $>1$, there were 32 peaks (20 peaks increased and 12 peaks decreased) in the tongue coating of patients with chronic gastritis by GC-TOF-MS analysis. 98 peaks (62 peaks increased and 36 peaks decreased) in the tongue coating of patients with chronic gastritis by UHPLC-QE-MS positive analysis, and 14 peaks (10 peaks increased and 4 peaks decreased) in the tongue coating of patients with chronic gastritis by UHPLCQE-MS negative analysis.

We used the criteria of similarity SV $>700$ or MS2 score $>0.6$, there were 19 peaks (11 peaks increased and 8 peaks decreased) in the tongue coating of patients with chronic gastritis by GC-TOF-MS analysis. 72 peaks (36 peaks increased and 36 peaks decreased) in the tongue coating of patients with chronic gastritis by UHPLC-QE-MS positive analysis. And 10 peaks (7 peaks increased and 3 peaks decreased) in the tongue coating of patients with chronic gastritis by UHPLC-QE-MS negative analysis.

\subsection{Different metabolites analysis}

As shown in Table 3, 85 metabolites were divided into 11 categories, of which the largest category was Lipids and lipid-like molecules, with 47 metabolites, Organic acids and derivatives with 13 metabolites, Organoheterocyclic compounds with 8 metabolites, Organic nitrogen compounds with 6 metabolites, Organic oxygen compounds with 4 metabolites, and Benzenoids with 2 metabolites, There was one metabolite in Homogeneous non-metal compounds, Nucleosides, nucleotides, and analogues, Phenylpropanoids and polyketides, Organosulfur compounds, and Hydrocarbons.

\subsection{Diagnostic model of chronic gastritis}

The diagnostic model was constructed by screening the different metabolites of tongue coating in 350 chronic gastritis patients and 50 healthy people. And the best diagnostic model was composed of 5 tongue coating metabolites. They were 1-methyladenosine and Sphinganine 1-phosphate screened by UHPLCQE-MS positive ion mode, 3-Hydroxycapric acid, 4-Ipomeanol, and Nervonic acid screened by UHPLC-QE-MS negative ion mode. Compared with healthy people, 
Sphinganine 1-phosphate, 4-Ipomeanol, and Nervonic acid were significantly upregulated in chronic gastritis patients, 1-methyladenosine and 3-Hydroxycapric acid were significantly downregulated in chronic gastritis patients. This diagnostic model had an accuracy of $95.4 \%$, a specificity of $87.4 \%$, and a sensitivity of $88.0 \%$.

\subsection{Correlation analysis between differential metabolites in tongue coating and gastroscopy indexes}

As can be seen from Figure 3-1, the differential metabolic markers of tongue coating obtained by GC-TOF-MS analysis between chronic gastritis group and healthy controls, octadecanol, and conduritol-beta-expoxide were negatively correlated with atrophic, and conduritol-beta-expoxide was negatively correlated with intestinal metaplasia. 6-deoxyglucitol was negatively correlated with bile reflux, and methylmaleic acid, 4-methylcatechol, and 2,4-dichloro-1-(2chloroethenyl)-benzene were negatively correlated with $\mathrm{Hp}$.

It can be seen from Figure 3-2 that UHPLC-QE-MS analyzes the differential metabolic markers of tongue coating obtained by comparing the chronic gastritis group with the healthy control group, including Trimethylaminoacetone, Sphinganine1-phosphate, alpha-Carboxy-delta-decalactone, and 5,6-Dihydroxyindole were positively correlated with intestinal metaplasia. Tetracosanoic acid, Lactosylceramide(d18:1/26:0), Chondrillasterol 3-[glucosyl-(1->4)-glucoside], Azelaic acid, and 1-Methyladenosine were negatively correlated with intestinal metaplasia. 3-benzoyloxy-11-oxo-12-ursen-28-oic acid was negatively correlated with gastric mucosal erosion. Sphinganine1-phosphate, alpha-Carboxy-delta-decalactone, and 5,6-Dihydroxyindole were positively correlated with atrophic. Tetracosanoic acid, Smilanippin A, Lactosylceramide(d18:1/26:0), Chondrillasterol 3-[glucosyl-(1->4)-glucoside], and Azelaic acid were negatively correlated with atrophic.

Table3 Identification of significant differential metabolites in tongue coating by comparison of chronic gastritis patients and healthy people. 


\section{Lipids and lipid-like molecules}

Arachidonic acid

$\begin{array}{llll}37.582 & 303 & 17.019 & 4.430\end{array}$

$<0.01 \quad 1.569 \quad 1.942$

UHPLC-

QE-MS

negative

ion

\begin{tabular}{|c|c|c|c|c|c|c|c|c|}
\hline Prostaglandin D2 & 46.555 & 351 & 1.937 & 1.098 & $<0.01$ & 1.657 & 0.819 & $\begin{array}{l}\text { UHPLC- } \\
\text { QE-MS } \\
\text { negative } \\
\text { ion }\end{array}$ \\
\hline 9,10-DHOME & 81.276 & 313 & 0.133 & 0.634 & $<0.01$ & 1.256 & -2.256 & $\begin{array}{l}\text { UHPLC- } \\
\text { QE-MS } \\
\text { negative } \\
\text { ion }\end{array}$ \\
\hline Azelaic acid & 359.713 & 187 & 0.191 & 0.796 & $<0.01$ & 1.731 & -2.062 & $\begin{array}{l}\text { UHPLC- } \\
\text { QE-MS } \\
\text { negative } \\
\text { ion }\end{array}$ \\
\hline Ethyl oleate & 41.892 & 309 & 0.692 & 0.332 & $<0.01$ & 1.241 & 1.060 & $\begin{array}{l}\text { UHPLC- } \\
\text { QE-MS } \\
\text { negative } \\
\text { ion }\end{array}$ \\
\hline 8,11,14-Eicosatrienoic acid & 37.965 & 305 & 0.760 & 0.229 & $<0.01$ & 1.364 & 1.727 & $\begin{array}{l}\text { UHPLC- } \\
\text { QE-MS } \\
\text { negative } \\
\text { ion }\end{array}$ \\
\hline Nervonic acid & 37.630 & 365 & 0.309 & 0.128 & $<0.01$ & 1.521 & 1.275 & $\begin{array}{l}\text { UHPLC- } \\
\text { QE-MS } \\
\text { negative } \\
\text { ion }\end{array}$ \\
\hline
\end{tabular}

\begin{tabular}{|c|c|c|c|c|c|c|c|c|}
\hline Tetracosanoic acid & 37.748 & 367 & 0.561 & 0.357 & $<0.01$ & 1.040 & 0.651 & $\begin{array}{l}\text { UHPLC- } \\
\text { QE-MS } \\
\text { negative } \\
\text { ion }\end{array}$ \\
\hline Foeniculoside VII & 71.454 & 349 & 0.517 & 1.452 & $<0.01$ & 1.168 & -1.490 & $\begin{array}{l}\text { UHPLC- } \\
\text { QE-MS } \\
\text { positive } \\
\text { ion }\end{array}$ \\
\hline
\end{tabular}

\begin{tabular}{|c|c|c|c|c|c|c|c|c|}
\hline Glycerol tripropanoate & 61.345 & 261 & 1.163 & 2.718 & $<0.01$ & 1.078 & -1.225 & $\begin{array}{l}\text { UHPLC- } \\
\text { QE-MS } \\
\text { positive } \\
\text { ion }\end{array}$ \\
\hline Solavetivone & 115.309 & 219 & 0.950 & 0.134 & $<0.01$ & 1.408 & 2.826 & $\begin{array}{l}\text { UHPLC- } \\
\text { QE-MS } \\
\text { positive } \\
\text { ion }\end{array}$ \\
\hline
\end{tabular}

\begin{tabular}{|c|c|c|c|c|c|c|c|c|}
\hline Patchoulenone & 61.062 & 219 & 11.219 & 3.010 & $<0.01$ & 1.438 & 1.898 & $\begin{array}{l}\text { UHPLC- } \\
\text { QE-MS } \\
\text { positive } \\
\text { ion }\end{array}$ \\
\hline Gibberellin A79 & 71.856 & 365 & 0.064 & 0.152 & $<0.01$ & 1.063 & -1.247 & $\begin{array}{l}\text { UHPLC- } \\
\text { QE-MS } \\
\text { positive } \\
\text { ion }\end{array}$ \\
\hline Oleic acid & 33.290 & 283 & 0.263 & 0.421 & $<0.01$ & 1.132 & -0.678 & $\begin{array}{l}\text { UHPLC- } \\
\text { QE-MS } \\
\text { positive } \\
\text { ion }\end{array}$ \\
\hline Dexamethasone & 77.202 & 393 & 0.262 & 1.323 & $<0.01$ & 1.249 & -2.335 & $\begin{array}{l}\text { UHPLC- } \\
\text { QE-MS } \\
\text { positive } \\
\text { ion }\end{array}$ \\
\hline 3-0-Methylniveusin A & 77.445 & 409 & 0.044 & 0.277 & $<0.01$ & 1.373 & -2.641 & $\begin{array}{l}\text { UHPLC- } \\
\text { QE-MS } \\
\text { positive } \\
\text { ion }\end{array}$ \\
\hline Hoduloside VII & 248.651 & 932 & 0.926 & 0.579 & $<0.01$ & 1.398 & 0.678 & $\begin{array}{l}\text { UHPLC- } \\
\text { QE-MS }\end{array}$ \\
\hline
\end{tabular}




\begin{tabular}{|c|c|c|c|c|c|c|c|c|}
\hline & & & & & & & & $\begin{array}{l}\text { positive } \\
\text { ion }\end{array}$ \\
\hline Fucoxanthin & 259.139 & 659 & 0.077 & 0.392 & $<0.01$ & 1.576 & -2.344 & $\begin{array}{l}\text { UHPLC- } \\
\text { QE-MS } \\
\text { positive } \\
\text { ion }\end{array}$ \\
\hline $\begin{array}{l}\text { N-Cyclopropyl-trans-2-cis-6- } \\
\text { nonadienamide }\end{array}$ & 46.068 & 194 & 0.174 & 0.114 & $<0.01$ & 1.364 & 0.611 & $\begin{array}{l}\text { UHPLC- } \\
\text { QE-MS } \\
\text { positive } \\
\text { ion }\end{array}$ \\
\hline 3-Hydroxyisovalerylcarnitine & 57.688 & 262 & 0.119 & 1.688 & $<0.01$ & 1.457 & -3.830 & $\begin{array}{l}\text { UHPLC- } \\
\text { QE-MS } \\
\text { positive } \\
\text { ion }\end{array}$ \\
\hline Lyciumoside III & 142.803 & 649 & 0.025 & 2.219 & $<0.01$ & 1.524 & -6.491 & $\begin{array}{l}\text { UHPLC- } \\
\text { QE-MS } \\
\text { positive } \\
\text { ion }\end{array}$ \\
\hline Eicosadienoic acid & 33.311 & 309 & 0.795 & 3.662 & $<0.01$ & 1.584 & -2.203 & $\begin{array}{l}\text { UHPLC- } \\
\text { QE-MS } \\
\text { positive } \\
\text { ion }\end{array}$ \\
\hline $\begin{array}{l}\text { 3beta-Acetoxy-11alpha-methoxy- } \\
\text { 12-ursen-28-oic acid }\end{array}$ & 193.697 & 543 & 0.092 & 0.044 & $<0.01$ & 1.400 & 1.071 & $\begin{array}{l}\text { UHPLC- } \\
\text { QE-MS } \\
\text { positive } \\
\text { ion }\end{array}$ \\
\hline Antibiotic X 14889C & 257.759 & 615 & 0.082 & 0.406 & $<0.01$ & 1.561 & -2.308 & $\begin{array}{l}\text { UHPLC- } \\
\text { QE-MS } \\
\text { positive } \\
\text { ion }\end{array}$ \\
\hline 2-Ethyl-2-Hydroxybutyric acid & 58.656 & 133 & 0.727 & 2.302 & $<0.01$ & 1.406 & -1.663 & $\begin{array}{l}\text { UHPLC- } \\
\text { QE-MS } \\
\text { positive } \\
\text { ion }\end{array}$ \\
\hline Palmitoyl glucuronide & 293.193 & 419 & 0.408 & 0.261 & $<0.01$ & 1.422 & 0.648 & $\begin{array}{l}\text { UHPLC- } \\
\text { QE-MS } \\
\text { positive } \\
\text { ion }\end{array}$ \\
\hline Physalin 0 & 314.512 & 529 & 0.129 & 0.082 & $<0.01$ & 1.426 & 0.663 & $\begin{array}{l}\text { UHPLC- } \\
\text { QE-MS } \\
\text { positive } \\
\text { ion }\end{array}$ \\
\hline Desglucocoroloside & 220.265 & 505 & 0.030 & 0.017 & $<0.01$ & 1.078 & 0.792 & $\begin{array}{l}\text { UHPLC- } \\
\text { QE-MS } \\
\text { positive } \\
\text { ion }\end{array}$ \\
\hline 3-Methylglutarylcarnitine & 84.851 & 290 & 0.257 & 0.182 & $<0.01$ & 1.289 & 0.502 & $\begin{array}{l}\text { UHPLC- } \\
\text { QE-MS } \\
\text { positive } \\
\text { ion }\end{array}$ \\
\hline Smilanippin A & 113.780 & 725 & 0.594 & 0.375 & $<0.01$ & 1.408 & 0.662 & $\begin{array}{l}\text { UHPLC- } \\
\text { QE-MS } \\
\text { positive } \\
\text { ion }\end{array}$ \\
\hline PS(16:1(9Z)/18:4(6Z,9Z,12Z,15Z)) & 207.222 & 754 & 0.069 & 0.573 & $<0.01$ & 1.592 & -3.047 & $\begin{array}{l}\text { UHPLC- } \\
\text { QE-MS } \\
\text { positive } \\
\text { ion }\end{array}$ \\
\hline $\mathrm{CPA}(18: 0 / 0: 0)$ & 278.918 & 421 & 0.210 & 0.131 & $<0.01$ & 1.368 & 0.682 & $\begin{array}{l}\text { UHPLC- } \\
\text { QE-MS } \\
\text { positive } \\
\text { ion }\end{array}$ \\
\hline Cucurbitacin I 2-glucoside & 205.358 & 677 & 1.712 & 1.092 & $<0.01$ & 1.092 & 0.648 & $\begin{array}{l}\text { UHPLC- } \\
\text { QE-MS } \\
\text { positive } \\
\text { ion }\end{array}$ \\
\hline Glycerol tributanoate & 125.367 & 303 & 0.170 & 0.563 & $<0.01$ & 1.081 & -1.728 & $\begin{array}{l}\text { UHPLC- } \\
\text { QE-MS } \\
\text { positive } \\
\text { ion }\end{array}$ \\
\hline Fasciculic acid C & 116.186 & 710 & 0.470 & 1.367 & $<0.01$ & 1.406 & -1.539 & UHPLC- \\
\hline
\end{tabular}




\begin{tabular}{|c|c|c|c|c|c|c|c|c|}
\hline & & & & & & & & $\begin{array}{l}\text { QE-MS } \\
\text { positive } \\
\text { ion }\end{array}$ \\
\hline Hebevinoside I & 38.382 & 809 & 0.097 & 0.365 & $<0.01$ & 1.375 & -1.917 & $\begin{array}{l}\text { UHPLC- } \\
\text { QE-MS } \\
\text { positive } \\
\text { ion }\end{array}$ \\
\hline 2,4,6-Octatriynoic acid & 440.442 & 133 & 0.296 & 0.478 & $<0.01$ & 1.127 & -0.692 & $\begin{array}{l}\text { UHPLC- } \\
\text { QE-MS } \\
\text { positive } \\
\text { ion }\end{array}$ \\
\hline Lycoperoside D & 154.407 & 740 & 0.568 & 11.651 & $<0.01$ & 1.352 & -4.358 & $\begin{array}{l}\text { UHPLC- } \\
\text { QE-MS } \\
\text { positive } \\
\text { ion }\end{array}$ \\
\hline $\begin{array}{l}\text { Chondrillasterol 3-[glucosyl-(1->4)- } \\
\text { glucoside] }\end{array}$ & 97.620 & 737 & 0.687 & 1.821 & $<0.01$ & 1.488 & -1.406 & $\begin{array}{l}\text { UHPLC- } \\
\text { QE-MS } \\
\text { positive } \\
\text { ion }\end{array}$ \\
\hline Physapruin B & 227.103 & 603 & 0.099 & 0.058 & $<0.01$ & 1.112 & 0.778 & $\begin{array}{l}\text { UHPLC- } \\
\text { QE-MS } \\
\text { positive } \\
\text { ion }\end{array}$ \\
\hline Traumatic acid & 281.345 & 229 & 0.497 & 0.421 & 0.047 & 1.196 & 0.239 & $\begin{array}{l}\text { UHPLC- } \\
\text { QE-MS } \\
\text { positive } \\
\text { ion }\end{array}$ \\
\hline Lactosylceramide (d18:1/26:0) & 31.388 & 1003 & 0.003 & 1.964 & $<0.01$ & 1.364 & -9.313 & $\begin{array}{l}\text { UHPLC- } \\
\text { QE-MS } \\
\text { positive } \\
\text { ion }\end{array}$ \\
\hline Sphinganine 1-phosphate & 51.580 & 382 & 0.166 & 0.069 & $<0.01$ & 1.554 & 1.256 & $\begin{array}{l}\text { UHPLC- } \\
\text { QE-MS } \\
\text { positive } \\
\text { ion }\end{array}$ \\
\hline $\begin{array}{l}\text { 3-Benzoyloxy-11-oxo-12-ursen-28- } \\
\text { oic acid }\end{array}$ & 34.377 & 575 & 2.140 & 1.439 & $<0.01$ & 1.278 & 0.573 & $\begin{array}{l}\text { UHPLC- } \\
\text { QE-MS } \\
\text { positive } \\
\text { ion }\end{array}$ \\
\hline Momordicoside $\mathrm{K}$ & 69.766 & 649 & 0.674 & 1.394 & $<0.01$ & 1.508 & -1.049 & $\begin{array}{l}\text { UHPLC- } \\
\text { QE-MS } \\
\text { positive } \\
\text { ion }\end{array}$ \\
\hline $\mathrm{PI}(22: 5(4 Z, 7 Z, 10 Z, 13 Z, 16 Z) / 16: 0)$ & 244.507 & 886 & 0.154 & 0.528 & $<0.01$ & 1.368 & -1.779 & $\begin{array}{l}\text { UHPLC- } \\
\text { QE-MS } \\
\text { positive } \\
\text { ion }\end{array}$ \\
\hline Vinaginsenoside R3 & 84.853 & 932 & 0.466 & 1.065 & $<0.01$ & 1.445 & -1.192 & $\begin{array}{l}\text { UHPLC- } \\
\text { QE-MS } \\
\text { positive } \\
\text { ion }\end{array}$ \\
\hline \multicolumn{9}{|l|}{ Organic acids and derivatives } \\
\hline glycolic acid & 5.611 & 147 & 0.001 & 0.002 & 0.005 & 1.229 & -0.518 & $\begin{array}{l}\text { GC-TOF- } \\
\text { MS }\end{array}$ \\
\hline pantothenic acid & 11.358 & 103 & 4.356E-04 & 2.233E-04 & $<0.01$ & 1.143 & 0.964 & $\begin{array}{l}\text { GC-TOF- } \\
\text { MS }\end{array}$ \\
\hline 3-Hydroxycapric acid & 83.306 & 187 & 0.214 & 0.441 & $<0.01$ & 1.383 & -1.043 & $\begin{array}{l}\text { UHPLC- } \\
\text { QE-MS } \\
\text { negative } \\
\text { ion }\end{array}$ \\
\hline Bortezomib & 285.056 & 407 & 0.080 & 0.811 & $<0.01$ & 1.026 & -3.350 & $\begin{array}{l}\text { UHPLC- } \\
\text { QE-MS } \\
\text { positive } \\
\text { ion }\end{array}$ \\
\hline Ustiloxin D & 287.091 & 495 & 0.044 & 0.450 & $<0.01$ & 1.239 & -3.339 & $\begin{array}{l}\text { UHPLC- } \\
\text { QE-MS } \\
\text { positive } \\
\text { ion }\end{array}$ \\
\hline Symmetric dimethylarginine & 158.848 & 203 & 4.835 & 6.288 & $<0.01$ & 1.032 & -0.379 & $\begin{array}{l}\text { UHPLC- } \\
\text { QE-MS }\end{array}$ \\
\hline
\end{tabular}




\begin{tabular}{|c|c|c|c|c|c|c|c|c|}
\hline & & & & & & & & $\begin{array}{l}\text { positive } \\
\text { ion }\end{array}$ \\
\hline Oseltamivir & 314.576 & 313 & 0.145 & 0.096 & $<0.01$ & 1.397 & 0.593 & $\begin{array}{l}\text { UHPLC- } \\
\text { QE-MS } \\
\text { positive } \\
\text { ion }\end{array}$ \\
\hline Arginyl-Arginine & 296.631 & 331 & 2.106 & 1.462 & 0.045 & 1.439 & 0.526 & $\begin{array}{l}\text { UHPLC- } \\
\text { QE-MS } \\
\text { positive } \\
\text { ion }\end{array}$ \\
\hline Arginyl-Histidine & 166.723 & 312 & 0.423 & 0.284 & 0.010 & 1.342 & 0.574 & $\begin{array}{l}\text { UHPLC- } \\
\text { QE-MS } \\
\text { positive } \\
\text { ion }\end{array}$ \\
\hline $\begin{array}{l}\text { Dihydro-3-(2-octenyl)-2,5- } \\
\text { furandione }\end{array}$ & 281.391 & 211 & 0.127 & 0.101 & 0.012 & 1.196 & 0.323 & $\begin{array}{l}\text { UHPLC- } \\
\text { QE-MS } \\
\text { positive } \\
\text { ion }\end{array}$ \\
\hline N-Acetylleucine & 265.067 & 174 & 0.154 & 0.099 & $<0.01$ & 1.231 & 0.632 & $\begin{array}{l}\text { UHPLC- } \\
\text { QE-MS } \\
\text { positive } \\
\text { ion }\end{array}$ \\
\hline Valyl-Isoleucine & 221.874 & 231 & 0.092 & 0.032 & $<0.01$ & 1.161 & 1.498 & $\begin{array}{l}\text { UHPLC- } \\
\text { QE-MS } \\
\text { positive } \\
\text { ion }\end{array}$ \\
\hline $\begin{array}{l}\text { L-cis-3-Amino-2- } \\
\text { pyrrolidinecarboxylic acid }\end{array}$ & 75.782 & 131 & 0.032 & 0.683 & $<0.01$ & 1.399 & -4.437 & $\begin{array}{l}\text { UHPLC- } \\
\text { QE-MS } \\
\text { positive } \\
\text { ion }\end{array}$ \\
\hline \multicolumn{9}{|l|}{ Organoheterocyclic compounds } \\
\hline 1-(1-Pyrrolidinyl)-2-propanone & 53.003 & 128 & 1.823 & 2.480 & $<0.01$ & 1.031 & -0.444 & $\begin{array}{l}\text { UHPLC- } \\
\text { QE-MS } \\
\text { positive } \\
\text { ion }\end{array}$ \\
\hline Pyrimidine & 112.505 & 81 & 0.097 & 0.320 & $<0.01$ & 1.275 & -1.728 & $\begin{array}{l}\text { UHPLC- } \\
\text { QE-MS } \\
\text { positive } \\
\text { ion }\end{array}$ \\
\hline 5,6-Dihydroxyindole & 45.066 & 150 & 0.251 & 0.169 & $<0.01$ & 1.128 & 0.574 & $\begin{array}{l}\text { UHPLC- } \\
\text { QE-MS } \\
\text { positive } \\
\text { ion }\end{array}$ \\
\hline $\begin{array}{l}\text { N1-Methyl-4-pyridone-3- } \\
\text { carboxamide }\end{array}$ & 79.822 & 153 & 0.212 & 0.176 & 0.032 & 1.002 & 0.269 & $\begin{array}{l}\text { UHPLC- } \\
\text { QE-MS } \\
\text { positive } \\
\text { ion }\end{array}$ \\
\hline 2-Methylpiperidine & 299.990 & 100 & 3.360 & 2.030 & $<0.01$ & 1.371 & 0.727 & $\begin{array}{l}\text { UHPLC- } \\
\text { QE-MS } \\
\text { positive } \\
\text { ion }\end{array}$ \\
\hline alpha-Carboxy-delta-decalactone & 125.959 & 215 & 0.080 & 0.336 & $<0.01$ & 1.087 & -2.065 & $\begin{array}{l}\text { UHPLC- } \\
\text { QE-MS } \\
\text { positive } \\
\text { ion }\end{array}$ \\
\hline I-Urobilin & 174.113 & 591 & 0.121 & 0.476 & $<0.01$ & 1.214 & -1.974 & $\begin{array}{l}\text { UHPLC- } \\
\text { QE-MS } \\
\text { positive } \\
\text { ion }\end{array}$ \\
\hline Garcimangosone C & 282.076 & 413 & 0.857 & 0.656 & 0.048 & 1.287 & 0.387 & $\begin{array}{l}\text { UHPLC- } \\
\text { QE-MS } \\
\text { positive } \\
\text { ion }\end{array}$ \\
\hline \multicolumn{9}{|l|}{ Organic nitrogen compounds } \\
\hline 1-Butylamine & 268.847 & 74 & 12.807 & 7.239 & $<0.01$ & 1.402 & 0.823 & $\begin{array}{l}\text { UHPLC- } \\
\text { QE-MS } \\
\text { positive } \\
\text { ion }\end{array}$ \\
\hline Nervonyl carnitine & 237.324 & 102 & 636.919 & 429.936 & $<0.01$ & 1.351 & 0.567 & $\begin{array}{l}\text { UHPLC- } \\
\text { QE-MS }\end{array}$ \\
\hline
\end{tabular}




\begin{tabular}{|c|c|c|c|c|c|c|c|c|}
\hline & & & & & & & & $\begin{array}{l}\text { positive } \\
\text { Ion }\end{array}$ \\
\hline Isobutylpropylamine & 231.650 & 116 & 0.444 & 0.294 & $<0.01$ & 1.294 & 0.596 & $\begin{array}{l}\text { UHPLC- } \\
\text { QE-MS } \\
\text { positive } \\
\text { ion }\end{array}$ \\
\hline 2-Diethylaminoethanol & 300.009 & 118 & 196.915 & 117.700 & $<0.01$ & 1.441 & 0.742 & $\begin{array}{l}\text { UHPLC- } \\
\text { QE-MS } \\
\text { positive } \\
\text { ion }\end{array}$ \\
\hline Dipyridamole & 47.761 & 253 & 0.366 & 9.287 & 0.016 & 1.060 & -4.666 & $\begin{array}{l}\text { UHPLC- } \\
\text { QE-MS } \\
\text { positive } \\
\text { ion }\end{array}$ \\
\hline L-Histidinol & 62.697 & 142 & 0.424 & 0.160 & $<0.01$ & 1.289 & 1.406 & $\begin{array}{l}\text { UHPLC- } \\
\text { QE-MS } \\
\text { positive } \\
\text { ion }\end{array}$ \\
\hline \multicolumn{9}{|l|}{ Organic oxygen compounds } \\
\hline glycerol & 7.006 & 85 & 1.092E-04 & $2.011 \mathrm{E}-04$ & $<0.01$ & 1.884 & -0.882 & $\begin{array}{l}\text { GC-TOF- } \\
\text { MS }\end{array}$ \\
\hline 4-Ipomeanol & 72.941 & 167 & 0.405 & 0.072 & $<0.01$ & 1.626 & 2.499 & $\begin{array}{l}\text { UHPLC- } \\
\text { QE-MS } \\
\text { negative } \\
\text { ion }\end{array}$ \\
\hline Leonuriside A & 372.037 & 333 & 0.012 & 0.235 & $<0.01$ & 1.365 & -4.293 & $\begin{array}{l}\text { UHPLC- } \\
\text { QE-MS } \\
\text { positive } \\
\text { ion }\end{array}$ \\
\hline Trimethylaminoacetone & 206.235 & 116 & 0.123 & 0.095 & $<0.01$ & 1.324 & 0.380 & $\begin{array}{l}\text { UHPLC- } \\
\text { QE-MS } \\
\text { positive } \\
\text { ion }\end{array}$ \\
\hline \multicolumn{9}{|l|}{ Benzenoids } \\
\hline Dictagymnin & 60.998 & 203 & 0.237 & 0.059 & $<0.01$ & 1.007 & 2.008 & $\begin{array}{l}\text { UHPLC- } \\
\text { QE-MS } \\
\text { positive } \\
\text { ion }\end{array}$ \\
\hline Salmeterol & 57.091 & 416 & 0.458 & 1.005 & $<0.01$ & 1.466 & -1.134 & $\begin{array}{l}\text { UHPLC- } \\
\text { QE-MS } \\
\text { positive } \\
\text { ion }\end{array}$ \\
\hline \multicolumn{9}{|c|}{ Homogeneous non-metal compounds } \\
\hline hydroxylamine & 5.968 & 85 & $6.208 \mathrm{E}-04$ & 0.001 & $<0.01$ & 1.240 & -0.834 & $\begin{array}{l}\text { GC-TOF- } \\
\text { MS }\end{array}$ \\
\hline \multicolumn{9}{|c|}{ Nucleosides, nucleotides, and analogues } \\
\hline 1-Methyladenosine & 185.868 & 282 & 0.375 & 0.692 & $<0.01$ & 1.015 & -0.884 & $\begin{array}{l}\text { UHPLC- } \\
\text { QE-MS } \\
\text { positive } \\
\text { ion }\end{array}$ \\
\hline \multicolumn{9}{|c|}{ Phenylpropanoids and polyketides } \\
\hline Sofalcone & 285.882 & 451 & 0.064 & 0.639 & $<0.01$ & 1.259 & -3.311 & $\begin{array}{l}\text { UHPLC- } \\
\text { QE-MS } \\
\text { positive } \\
\text { ion }\end{array}$ \\
\hline \multicolumn{9}{|l|}{ Organosulfur compounds } \\
\hline$(\hat{A} \pm)-2-P e n t a n e t h i o l$ & 32.769 & 105 & 0.419 & 0.279 & $<0.01$ & 1.195 & 0.590 & $\begin{array}{l}\text { UHPLC- } \\
\text { QE-MS } \\
\text { positive } \\
\text { ion }\end{array}$ \\
\hline \multicolumn{9}{|l|}{ Hydrocarbons } \\
\hline 1-Methyl-1,3-cyclohexadiene & 32.571 & 95 & 0.476 & 0.315 & $<0.01$ & 1.290 & 0.595 & $\begin{array}{l}\text { UHPLC- } \\
\text { QE-MS } \\
\text { positive } \\
\text { ion }\end{array}$ \\
\hline
\end{tabular}




\begin{tabular}{|c|c|c|c|c|c|c|c|c|}
\hline 6-deoxyglucitol & 10.173 & 117 & $1.979 \mathrm{E}-04$ & $1.416 \mathrm{E}-04$ & $<0.01$ & 2.679 & 0.483 & $\begin{array}{l}\text { GC-TOF- } \\
\text { MS }\end{array}$ \\
\hline myristic acid & 10.642 & 117 & 9.197E-04 & 0.002 & $<0.01$ & 1.264 & -1.213 & $\begin{array}{l}\text { GC-TOF- } \\
\text { MS }\end{array}$ \\
\hline octadecanol & 12.158 & 327 & $\begin{array}{l}2.58849 \mathrm{E}- \\
05\end{array}$ & $\begin{array}{l}3.63256 \mathrm{E}- \\
05\end{array}$ & $<0.01$ & 1.036 & -0.489 & $\begin{array}{l}\text { GC-TOF- } \\
\text { MS }\end{array}$ \\
\hline 1,2,4-benzenetriol & 9.344 & 124 & 0.006 & 0.003 & $<0.01$ & 2.238 & 0.835 & $\begin{array}{l}\text { GC-TOF- } \\
\text { MS }\end{array}$ \\
\hline threitol & 8.562 & 191 & $\begin{array}{l}4.8463 \mathrm{E}- \\
05\end{array}$ & $1.651 \mathrm{E}-04$ & $<0.01$ & 1.286 & -1.769 & $\begin{array}{l}\text { GC-TOF- } \\
\text { MS }\end{array}$ \\
\hline $\begin{array}{l}\text { 2,4-dichloro-1-(2-chloroethenyl)- } \\
\text { benzene }\end{array}$ & 7.925 & 170 & 0.012 & 0.008 & 0.011 & 2.553 & 0.645 & $\begin{array}{l}\text { GC-TOF- } \\
\text { MS }\end{array}$ \\
\hline asparagine dehydrated & 8.628 & 110 & $2.811 \mathrm{E}-04$ & $2.228 \mathrm{E}-04$ & $<0.01$ & 1.493 & 0.335 & $\begin{array}{l}\text { GC-TOF- } \\
\text { MS }\end{array}$ \\
\hline conduritol-beta-expoxide & 11.541 & 103 & 0.005 & 0.003 & $<0.01$ & 1.674 & 0.696 & $\begin{array}{l}\text { GC-TOF- } \\
\text { MS }\end{array}$ \\
\hline methylmaleic acid & 7.923 & 170 & 0.012 & 0.008 & 0.037 & 2.782 & 0.601 & $\begin{array}{l}\text { GC-TOF- } \\
\text { MS }\end{array}$ \\
\hline 4-methylcatechol & 7.928 & 170 & 0.012 & 0.008 & 0.036 & 2.120 & 0.607 & $\begin{array}{l}\text { GC-TOF- } \\
\text { MS }\end{array}$ \\
\hline glucose-6-phosphate major & 12.806 & 111 & $\begin{array}{l}5.8939 \mathrm{E}- \\
05\end{array}$ & $\begin{array}{l}4.41292 \mathrm{E}- \\
05\end{array}$ & $<0.01$ & 2.808 & 0.417 & $\begin{array}{l}\text { GC-TOF- } \\
\text { MS }\end{array}$ \\
\hline uridine minor & 13.354 & 145 & 0.002 & 0.001 & 0.015 & 2.825 & 0.387 & $\begin{array}{l}\text { GC-TOF- } \\
\text { MS }\end{array}$ \\
\hline methylmalonic acid & 6.698 & 85 & $\begin{array}{l}7.14578 \mathrm{E}- \\
05\end{array}$ & 1.287E-04 & $<0.01$ & 1.764 & -0.849 & $\begin{array}{l}\text { GC-TOF- } \\
\text { MS }\end{array}$ \\
\hline 5-hydroxynorvaline & 8.88 & 85 & $9.138 \mathrm{E}-04$ & 0.002 & $<0.01$ & 1.157 & -0.977 & $\begin{array}{l}\text { GC-TOF- } \\
\text { MS }\end{array}$ \\
\hline sulfuric acid & 6.326 & 117 & $1.023 \mathrm{E}-04$ & $\begin{array}{l}7.39354 \mathrm{E}- \\
05\end{array}$ & $<0.01$ & 1.921 & 0.469 & $\begin{array}{l}\text { GC-TOF- } \\
\text { MS }\end{array}$ \\
\hline (10S,11R)-Pterosin C 4-glucoside & 282.232 & 397 & 15.388 & 11.382 & 0.024 & 1.345 & 0.435 & $\begin{array}{l}\text { UHPLC- } \\
\text { QE-MS } \\
\text { positive } \\
\text { ion }\end{array}$ \\
\hline
\end{tabular}

\subsection{Change trends of differential metabolite in different pathological stages}

Table 4 The content of metabolites in the tongue coating of chronic gastritis patients at different pathological stages and healthy people. 


\begin{tabular}{|c|c|c|c|c|}
\hline Metabolites & Mild & Moderate & Severe & Healthy controls \\
\hline \multicolumn{5}{|l|}{ atrophic } \\
\hline octadecanol & $1.534 \mathrm{E}-05$ & 1.045E-05 & 1.994E-05 & 3.63256E-05 \\
\hline conduritol-beta-expoxide & $4.46 \mathrm{E}-03$ & $3.17 \mathrm{E}-03^{*}$ & $4.66 \mathrm{E}-03$ & $2.89 \mathrm{E}-03$ \\
\hline Tetracosanoic acid & 5.98E-01 & 5.27E-01 & 5.57E-01 & 3.57E-01 \\
\hline Sphinganine 1-phosphate & 1.71E-01 & 1.60E-01 & 1.17E-01 & $6.93 \mathrm{E}-02$ \\
\hline Smilanippin A & 5.64E-01 & 5.67E-01 & $6.73 \mathrm{E}-01$ & $3.75 \mathrm{E}-01$ \\
\hline Lactosylceramide (d18:1/26:0) & 3.17E-03 & 3.19E-03 & 2.68E-03 & $1.96 \mathrm{E}+00$ \\
\hline Chondrillasterol 3-[glucosyl-(1->4)-glucoside] & $6.59 \mathrm{E}-01$ & $6.46 \mathrm{E}-01$ & 7.64E-01 & $1.82 \mathrm{E}+00$ \\
\hline Azelaic acid & 1.88E-01 & 1.85E-01 & $1.70 \mathrm{E}-01$ & $7.96 \mathrm{E}-01$ \\
\hline alpha-Carboxy-delta-decalactone & 7.91E-02 & 8.74E-02 & 8.03E-02 & $3.36 \mathrm{E}-01$ \\
\hline 5,6-Dihydroxyindole & $2.55 \mathrm{E}-01$ & $2.55 \mathrm{E}-01$ & $2.48 \mathrm{E}-01$ & $1.69 \mathrm{E}-01$ \\
\hline \multicolumn{5}{|l|}{ intestinal metaplasia } \\
\hline conduritol-beta-expoxide & $4.62 \mathrm{E}-03$ & $3.20 \mathrm{E}-03^{*}$ & $4.66 \mathrm{E}-03$ & $2.89 \mathrm{E}-03$ \\
\hline trimethylaminoacetone & $1.24 \mathrm{E}-01$ & 1.16E-01 & $1.12 \mathrm{E}-01$ & $9.46 \mathrm{E}-02$ \\
\hline tetracosanoic acid & $6.08 \mathrm{E}-01$ & $5.24 \mathrm{E}-01$ & 5.57E-01 & $3.57 \mathrm{E}-01$ \\
\hline Sphinganine 1-phosphate & 1.64E-01 & $1.58 \mathrm{E}-01$ & 1.17E-01 & $6.93 \mathrm{E}-02$ \\
\hline Lactosylceramide (d18:1/26:0) & $3.51 \mathrm{E}-03$ & $3.34 \mathrm{E}-03$ & $2.68 \mathrm{E}-03$ & $1.96 \mathrm{E}+00$ \\
\hline Chondrillasterol 3-[glucosyl-(1->4)-glucoside] & $6.75 \mathrm{E}-01$ & $6.56 \mathrm{E}-01$ & 7.64E-01 & $1.82 \mathrm{E}+00$ \\
\hline Azelaic acid & $1.84 \mathrm{E}-01$ & $1.85 \mathrm{E}-01$ & $1.70 \mathrm{E}-01$ & 7.96E-01 \\
\hline alpha-Carboxy-delta-decalactone & $8.14 \mathrm{E}-02$ & 8.77E-02 & 8.03E-02 & $3.36 \mathrm{E}-01$ \\
\hline 5,6-Dihydroxyindole & $2.54 \mathrm{E}-01$ & $2.56 \mathrm{E}-01$ & 2.48E-01 & $1.69 \mathrm{E}-01$ \\
\hline 1-Methyladenosine & 3.60E-01 & 3.92E-01 & 4.52E-01 & $6.92 \mathrm{E}-01$ \\
\hline \multicolumn{5}{|l|}{ Hp } \\
\hline methylmaleic acid & 1.17E-02 & 1.19E-02 & $1.22 \mathrm{E}-02$ & 8.13E-03 \\
\hline 4-methylcatechol & 1.03E-02 & 1.13E-02 & $1.22 \mathrm{E}-02$ & $7.82 \mathrm{E}-03$ \\
\hline 2,4-dichloro-1-(2-chloroethenyl)-benzene & 1.17E-02 & 1.13E-02 & $1.22 \mathrm{E}-02$ & 7.79E-03 \\
\hline
\end{tabular}

* There were differences between moderate and mild, $P<0.05$.

According to Figure 4-1 to Figure 4-3 and Table 4 we can see that in the tongue coating of atrophic, the contents of octadecanol, Lactosylceramide (d18:1/26:0), Chondrillasterol 3-[glucosyl-(1->4)-glucoside], Azelaic acid, and alpha-Carboxy-delta-decalactone in the three stages of atrophic were lower than those in the healthy control group. The contents of conduritol-beta-expoxide, Tetracosanoic acid, Sphinganine 1-phosphate, Smilanippin A and 5,6Dihydroxyindole in the three stages of atrophic were higher than those in the healthy control group. The content of conduritol-beta-expoxide decreased significantly in mild atrophic compared with moderate atrophic $(P<0.05)$. The changing trend of alpha-Carboxy-delta-decalactone content in the tongue coatings of atrophic patients was first increased and then decreased. The changing trend of octadecanol, conduritol-beta-expoxide, Tetracosanoic acid, and Chondrillasterol 3-[glucosyl-(1->4)-glucoside] contents in the tongue coatings of atrophic patients was first decreased and then increased. The content of Smilanippin A in the tongue coating of atrophic patients showed an upward trend. The contents of Sphinganine 1-phosphate, Lactosylceramide (d18:1/26:0), Azelaic acid, and 5,6-Dihydroxyindole in the tongue coating of atrophic patients showed a downward trend.

In the tongue coating of intestinal metaplasia patients, the contents of Lactosylceramide (d18:1/26:0), Chondrillasterol 3-[glucosyl-(1->4)-glucoside], Azelaic acid, alpha-Carboxy-delta-decalactone, and 1-Methyladenosine in the three stages of intestinal metaplasia were lower than those in the healthy control group. The contents of conduritol-beta-expoxid, trimethylaminoacetone, tetracosanoic acid, Sphinganine 1-phosphate, and 5,6-Dihydroxyindole in the three stages of intestinal metaplasia were higher than those in the healthy control group. The content of conduritol-beta-expoxide decreased significantly in mild intestinal metaplasia compared with moderate intestinal metaplasia $(P<0.05)$. The changing trend of Azelaic acid, alpha-Carboxy-delta-decalactone, and 5,6Dihydroxyindole contents in the tongue coatings of intestinal metaplasia patients was first increased and then decreased. The changing trend of conduritolbeta-expoxide, tetracosanoic acid, and Chondrillasterol 3-[glucosyl-(1->4)-glucoside] contents in the tongue coatings of intestinal metaplasia patients was first decreased and then increased. The content of 1-Methyladenosine in the tongue coating of intestinal metaplasia patients showed an upward trend. The contents of trimethylaminoacetone, Sphinganine 1-phosphate, Lactosylceramide (d18:1/26:0) in the tongue coating of intestinal metaplasia patients showed a downward trend. 
In the tongue coating of $\mathrm{Hp}$ patients, the contents of methylmaleic acid, 4-methylcatechol, and 2,4-dichloro-1-(2-chloroethenyl)-benzene in the three stages of $\mathrm{Hp}$ were higher than those in the healthy control group. The contents of Methylmaleic acid and 4-methylcatechol in the tongue coating of Hp infection patients showed an upward trend. The changing trend of 2,4-dichloro-1-(2-chloroethenyl)-benzene content in the tongue coatings of Hp infection patients was first decreased and then increased.

\section{Discussion}

In this study, we used UHPC-QE-MS and GC-TOF-MS to detect the metabolites in the tongue coating of 350 patients with chronic gastritis, established the diagnostic model of the screened differential metabolites, and analyzed the correlation between the detected differential metabolites and the results of gastroscopic pathological indexes, and we explored the variation pattern of differential metabolite levels according to the degree of chronic gastritis lesions.

It can be seen from the metabolomic analysis of chronic gastritis tongue coating in Table 3, compared with the healthy control group, the number of differential metabolites of Lipids and lipid-like molecules in the tongue coating of chronic gastritis patients was the largest (there were 47 substances in total, of which 22 substances were upregulated and 25 substances were downregulated), accounting for $55.29 \%$ of the total metabolites, suggesting that Lipids and lipid-like molecules were the main metabolic disorder in patients with chronic gastritis. Some researchers have also found that Lipids and lipid-like molecules were the main differential metabolites in the serum of patients with chronic gastritis by using the method of serum metabolomics, and believe that Lipids and lipid-like molecules may promote the development of chronic gastritis to gastric cancer [13].

Among the differential metabolites of Lipids and lipid-like molecules there is a substance called Sphinganine 1-phosphate, which is significantly higher than that of healthy people. Sphinganine 1-phosphate is one of the markers of the diagnostic model of chronic gastritis. It has also been found to be significantly positively correlated with atrophic and intestinal metaplasia. In addition, we also found that although the content of Sphinganine 1-phosphate in patients with gastric mucosal atrophic and intestinal metaplasia was always significantly higher than that in healthy controls, the level of Sphinganine 1-phosphate decreased gradually with the aggravation of the severity of gastric mucosal atrophic and intestinal metaplasia in patients with gastritis. This is a very interesting phenomenon, and its mechanism needs to be further discussed. Other studies have also shown that Sphinganine 1-phosphate was related to the occurrence of cancer, which can promote tumor growth and metastasis [14-15]. Among the differential metabolites screened by the diagnostic model of chronic gastritis, except Sphinganine 1-phosphate, there was another substance belonging to the differential metabolites of Lipids and lipid-like molecules, which was Nervonic acid. Some researchers found Nervonic acid in the mucosa of patients with gastric and colon cancer [16]. It can be seen that nervonic acid exists not only in the upper digestive tract, but also in the lower digestive tract.

In our study on the correlation between differential metabolites in tongue coatings and pathological indexes in patients with chronic gastritis, we found that the number of differential metabolites of Lipids and lipid-like molecules was the largest, they were Sphinganine1-phosphate, Tetracosanoic acid, Smilanippin A, Lactosylceramide(d18:1/26:0), Chondrillasterol 3-[glucosyl-(1->4)-glucoside], Azelaic acid and 3-Benzoyloxy-11-oxo-12-ursen-28-oic acid.

In Figure 3-<link rid="fig3">1</link>-e and 3-<ink rid="fig3"> 1</link>-f, we found that the content of Smilanippin A in the tongue coating of patients with severe atrophic was higher than that of patients with mild and moderate atrophic, and the content of this metabolite in the tongue coating of patients with different degrees of atrophic was higher than that of healthy people. The content of Lactosylceramide (d18:1/26:0) in the tongue coating of patients with severe atrophic was lower than that of patients with mild atrophic and patients with moderate atrophic, and the content of this metabolite in the tongue coating of patients with different degrees of atrophic was lower than that of healthy people. In addition, as shown in figure 3-2-e, the content of Lactosylceramide (d18:1/26:0) gradually decreases with the aggravation of intestinal metaplasia, and the content in the three stages of intestinal metaplasia was lower than that in the tongue coating of healthy people. Studies have found that Lactosylceramide can promote the proliferation of colon cancer cells [17], and researchers have found that this substance exists in the serum of children with inflammatory bowel disease [18]. It can be seen that this substance will aggravate digestive system diseases. In Figure 3-1-g and 3-2-f, the content of Chondrillasterol 3-[glucosyl-(1->4)-glucoside] in the tongue coating of patients with severe atrophic and intestinal metaplasia was higher than that of patients with mild and moderate atrophic and intestinal metaplasia. Moreover, the content of this substance in the three stages of atrophic and intestinal metaplasia was lower than that in the tongue coating of healthy people.

In Figure 3-1-h and 3-2-g, the content of Azelaic acid gradually decreases with the aggravation of atrophic, and its content in the tongue coating of subjects with different degrees of atrophic was lower than that of healthy people. Moreover, the content of Azelaic acid in severe intestinal metaplasia subjects was lower than that in mild and moderate patients, and the content in the three stages of intestinal metaplasia was lower than that in the tongue coating of healthy people.

Organic acids and derivatives were the second largest metabolites in the tongue coating of patients with chronic gastritis. There were 13 metabolites ( 7 metabolites were upregulated and 6 metabolites were downregulated), among which pantothenic acid was upregulated. Some researchers have found that this substance was a potential biomarker of gastric cancer and was of great significance for the diagnosis of gastric cancer [19]. The differential metabolite 3Hydroxycapric acid screened by the diagnostic model of chronic gastritis also belongs to Organic acids and derivatives.

Other metabolites include Organoheterocyclic compounds (8 metabolites, 4 substances upregulated and 4 substances downregulated), Organic nitrogen compounds ( 6 metabolites, 5 substances upregulated and 1 substance downregulated), and Organic oxygen compounds (4 metabolites, 2 substances upregulated and 2 substances downregulated), Benzenoids (there are 2 metabolites, 1 upregulated and 1 downregulated), Homogeneous non-metal compounds, Nucleosides, nucleotides, and analogues, Phenylpropanoids and polyketides, Organosulfur compounds, and Hydrocarbons each have 1 metabolite. In these metabolic species, L-histidinol expression was upregulated. Some studies have shown that histidinol has the effect of reducing gastric acid and reducing gastrointestinal pain. In addition, it can also inhibit the proliferation and migration of lung cancer cells, thus play an anti-tumor role [20-21]. Dictagymnin expression was upregulated, and this substance has anti-inflammatory and tumor growth inhibitory effects [22]. It can be shown that patients with chronic gastritis were probably also undergoing self-repair by the body during their illness. From the correlation between chronic gastritis and 
pathological indexes, we found that the differential metabolites belonging to Organoheterocyclic compounds were alpha-Carboxy-delta-decalactone and 5,6Dihydroxyindole. In figure 3-1-i and 3-2-h, we can see that the content of alpha-Carboxy-delta-decalactone in the tongue coating of patients with moderate atrophic and intestinal metaplasia was higher than that of patients with mild and severe atrophic, and the content of this substance in the tongue coating of patients with different degrees of atrophic and intestinal metaplasia was lower than that of healthy people. In figure 3-1-j and 3-2-i, the content of 5,6Dihydroxyindole in the tongue coating of patients with severe atrophic and intestinal metaplasia was lower than that of patients with mild and moderate atrophic. Moreover, the content of this substance in the tongue coating of patients with different degrees of atrophic and intestinal metaplasia was higher than that of healthy people.

Among the differential metabolites screened by the diagnostic model of chronic gastritis, 4-Ipomeanol belongs to Organic oxygen compounds, and 1Methyladenosine belongs to Nucleosides, nucleotides, and analogues. 1-Methylladenosine was also found in the results of correlation of pathological indexes. In the trend figures of chronic gastritis with intestinal transformation, the content of 1-Methylladenosine increased with the increase of intestinal degree, but the content in the three stages of intestinal transformation was lower than that of healthy people.

In addition, we also found three substances in Figure 3-3 of the Hp trend figure: methylmaleic acid, 4-methylcatechol and 2,4-dichloro-1-(2-chloroethenyl)benzene. The contents of methylmaleic acid and 4-methylcatechol gradally increased with the aggravation of Hp. The content of 2,4-dichloro-1-(2chloroethenyl)-benzene in the tongue coating of moderate intestinal metaplasia patients was lower than that of mild and severe patients. Experiments on rats showed that 4-methylcatechol was correlated with the occurrence of gastric cancer [23]. While Hp infection was considered a risk factor for gastric cancer [24]. We suggest that 4-methylcatechol might be involved in the transformation from chronic gastritis with $\mathrm{Hp}$ infection to gastric cancer. In Table 4, we can also found that the content of conduritol-beta-expoxide decreases significantly with the aggravation of atrophic and intestinal metaplasia. However, we have not found the content change of this substance in digestive system diseases.

This study also has the following shortcomings. First, the proportion of sample cases in chronic gastritis group and healthy control group is large. Second, the age difference between chronic gastritis group and healthy control group is large. Although our previous study showed that the significant difference between the number of sample cases and age ratio between groups had a minor effect on the altered results of metabolite content in the tongue coating of patients with gastric precancerous lesions [25], in future studies, we will narrow the difference of sample cases and age between groups as much as possible. Last, this study only conducted a cross-sectional study of 350 patients with chronic gastritis. In the follow-up study, we will conduct a cohort study to further verify our current findings.

\section{Conclusions}

This study found that Lipids and lipid-like molecules were the main differential metabolites in the tongue coating of patients with chronic gastritis. Among these lipid metabolites, Sphinganine 1-phosphate was one of the metabolites to establish the diagnostic model of chronic gastritis. Its content change was significantly positively correlated with the occurrence of atrophic and intestinal metaplasia, but the level of this metabolite decreases with the aggravation of atrophic and intestinal metaplasia. Therefore, we believe that the change of Sphinganine 1-phosphate content in tongue coating has a certain correlation with the pathological progress of chronic gastritis, but its specific mechanism needs to be further discussed. We also found that conduritol-beta-expoxide was negatively correlated with atrophic and intestinal metaplasia, and its content decreased significantly with the aggravation of atrophic and intestinal metaplasia. Other researchers have not reported the above results. In future studies, we will further verify the changes of these tongue coating differential metabolites in patients with gastritis through cohort studies. We believe that in the future, these differential markers in tongue coating may help us to establish a noninvasive and convenient method to detect chronic gastritis and gastric precancerous lesion.

\section{Abbreviations}

TCM: Traditional Chinese Medicine

UPLC-MS: Ultra-performance liquid chromatography and mass spectrometry

LC-MS: Liquid chromatography and mass spectrometry

GC-TOF-MS: Gas chromatography-time-of-flight-mass spectroscopy

UHPLC-QE-MS: Liquid chromatography-Q exactive orbitrap-mass spectroscopy

Hp: Helicobacter pylori

BMl: Body mass index

EP: Eppendorf

QC: Quality control

PCA: Principal components analysis

OPLS-DA: Orthogonal projections to latent structures-discriminant analysis

FDR: False discovery rate

Page $15 / 24$ 
VIP: Variable importance in the projection

SV: Similarity value

FC: Fold change

CORR: Correlation coefficient

\section{Declarations}

\section{Funding}

This paper was funded by the National Natural Science Foundation of China (No. 82174279; No. 81703982). This work was also supported by Shanghai Biotree Biotech Co. Ltd.

\section{Conflicts of Interest}

The authors declare that the research was conducted in the absence of financial relationships or any commercial.

\section{Acknowledgments}

This work was also supported by Shanghai Biotree Biotech Co. Ltd.

\section{Consent to publish}

All authors agree to publish this article.

\section{Ethics approval and consent to participate}

This study was approved by the Ethics Committee of Shanghai University of TCM in China in December 2018 and performed in accordance with the Declaration of Helsinki. All the subjects had signed informed consent agreements before collecting samples.

\section{Authors' contributions}

Yiming Hao was responsible for the study. Yifeng Xu wrote the manuscript. Renling Zhang helped collect clinical samples. Junhong Lu helped revise the text and grammar of the manuscript. Yiqin Wang helped with the ideas of the study. Zhujing Zhu helped with the experimentation. All authors of this study were agreed to be accountable for all aspects of the work.

\section{Availability of data and materials}

The Ethics Committee of Shanghai University of Traditional Chinese Medicine limited the measurement data used to support the results of this study in order to protect the privacy of patients. For researchers who meet the criteria for obtaining confidential data, the data of this study can be obtained from Yiming Hao (Email: hymjj888@163.com).

\section{References}

[1] Sipponen P, Maaroos HI. Chronic gastritis. Scand J Gastroenterol, 2015, 50(6): 657-667

[2] Cui J, Cui H, Yang M, et al. Tongue coating microbiome as a potential biomarker for gastritis including precancerous cascade. Protein Cell. 2019 Jul;10(7):496-509

[3] Li FF, Zhao J, Qian P, et al. Metabolite changes in the greasy tongue coating of patients with chronic gastritis. Zhong Xi Yi Jie He Xue Bao. 2012,10(7):75765

[4] Sun ZM, Zhao J, Qian P, et al. Metabolic markers and microecological characteristics of tongue coating in patients with chronic gastritis. BMC Complement Altern Med. 2013,13:227

[5] Zeki ÖC, Eylem CC, Reçber T, et al. Integration of GC-MS and LC-MS for untargeted metabolomics profiling. J Pharm Biomed Anal. 2020 Oct 25; $190: 113509$

[6] Dixon MF, Genta RM, Yardley JH, et al. Classification and grading of gastritis. The updated Sydney System. International Workshop on the Histopathology of Gastritis, Houston 1994. Am J Surg Pathol. 1996,20(10):1161-81

[7] National Health and Family Planning Commission of PRC. Criteria of weight for adults. Vol. 2. Beijing, China: Standards Press of China; 2013

[8] Kind T, Wohlgemuth G, Lee DY, et al. FiehnLib: mass spectral and retention index libraries for metabolomics based on quadrupole and time-of-flight gas chromatography/mass spectrometry. Anal Chem. 2009,81(24):10038-10048

[9] Dunn WB, Broadhurst D, Begley P, et al. Procedures for large-scale metabolic profiling of serum and plasma using gas chromatography and liquid chromatography coupled to mass spectrometry. Nat Protoc. 2011,6(7):1060-1083 
[10] Smith CA, Want EJ, O'Maille G, et al. XCMS: processing mass spectrometry data for metabolite profiling using nonlinear peak alignment, matching, and identification. Anal Chem. 2006,78(3):779-787

[11] Yang C, Hao R, Du X, et al. GC-TOF/MS-based metabolomics studies on the effect of protein sources in formulated diet for pearl oyster Pinctada fucata martensii. Aquaculture. 2018,486:139-147

[12] Wang W, Zhao L, He Z, et al. Metabolomics-based evidence of the hypoglycemic effect of Ge-Gen-JiaoTai-Wan in type 2 diabetic rats via UHPLC-QTOF/MS analysis. J Ethnopharmacol. 2018,219:299-318

[13] Yu L, Lai Q, Feng Q, et al. Serum Metabolic Profiling Analysis of Chronic Gastritis and Gastric Cancer by Untargeted Metabolomics. Front Oncol. 2021 Mar $11 ; 11: 636917$

[14] Plano D, Amin S, Sharma AK. Importance of sphingosine kinase (SphK) as a target in developing cancer therapeutics and recent developments in the synthesis of novel SphK inhibitors. J Med Chem. 2014 Jul 10;57(13):5509-24

[15] Schneider G. S1P Signaling in the Tumor Microenvironment. Adv Exp Med Biol. 2020; 1223:129-153

[16] Pacuszka T, Jóźwiak W, Miller-Podraza H, et al. Neutral glycolipid composition in human cancer. Cancer Biochem Biophys. 1980;5(1):1-6

[17] Machala M, Procházková J, Hofmanová J, et al. Colon Cancer and Perturbations of the Sphingolipid Metabolism. Int J Mol Sci. 2019 Nov 30;20(23):6051

[18] Filimoniuk A, Blachnio-Zabielska A, Imierska M, et al. Sphingolipid Analysis Indicate Lactosylceramide as a Potential Biomarker of Inflammatory Bowel Disease in Children. Biomolecules. 2020 Jul 21;10(7):1083

[19] Jiang W, Zhou L, Lin S, et al. Metabolic profiles of gastric cancer cell lines with different degrees of differentiation. Int J Clin Exp Pathol. 2018,11(2):869-87

[20] Zu GX, Sun QQ, Chen J, et al. Urine metabolomics of rats with chronic atrophic gastritis. PLoS One. 2020 Nov 11;15(11): e0236203

[21] Escobar-Reséndiz R, Reyes-Esparza J, Blake IO, et al. Evaluation of antitumoral effect of the combination of L-histidine methyl esther hydrochloride of anfotericin B with antineoplastics on A549 cells. FASEB J. 2020;34: 1

[22] Wang JY, Wang Z, Li MY, et al. Dictamnine promotes apoptosis and inhibits epithelial-mesenchymal transition, migration, invasion and proliferation by downregulating the HIF-1a and Slug signaling pathways. Chem Biol Interact. 2018 Dec 25; 296:134-144

[23] Asakawa E, Hirose M, Hagiwara A, et al. Carcinogenicity of 4-methoxyphenol and 4-methylcatechol in F344 rats. Int J Cancer. 1994 Jan 2;56(1):146-52

[24] Malfertheiner P, Megraud F, O'Morain CA, et al. Management of Helicobacter pylori infection-the Maastricht IV/ Florence Consensus Report. Gut. 2012 May;61(5):646-64

[25] Hao Y, Zhang R, Morris R, et al. Metabolome and microbiome alterations in tongue coating of gastric precancerous lesion patients. Expert Rev Gastroenterol Hepatol. 2021 Aug;15(8):949-963

\section{Figures}


a
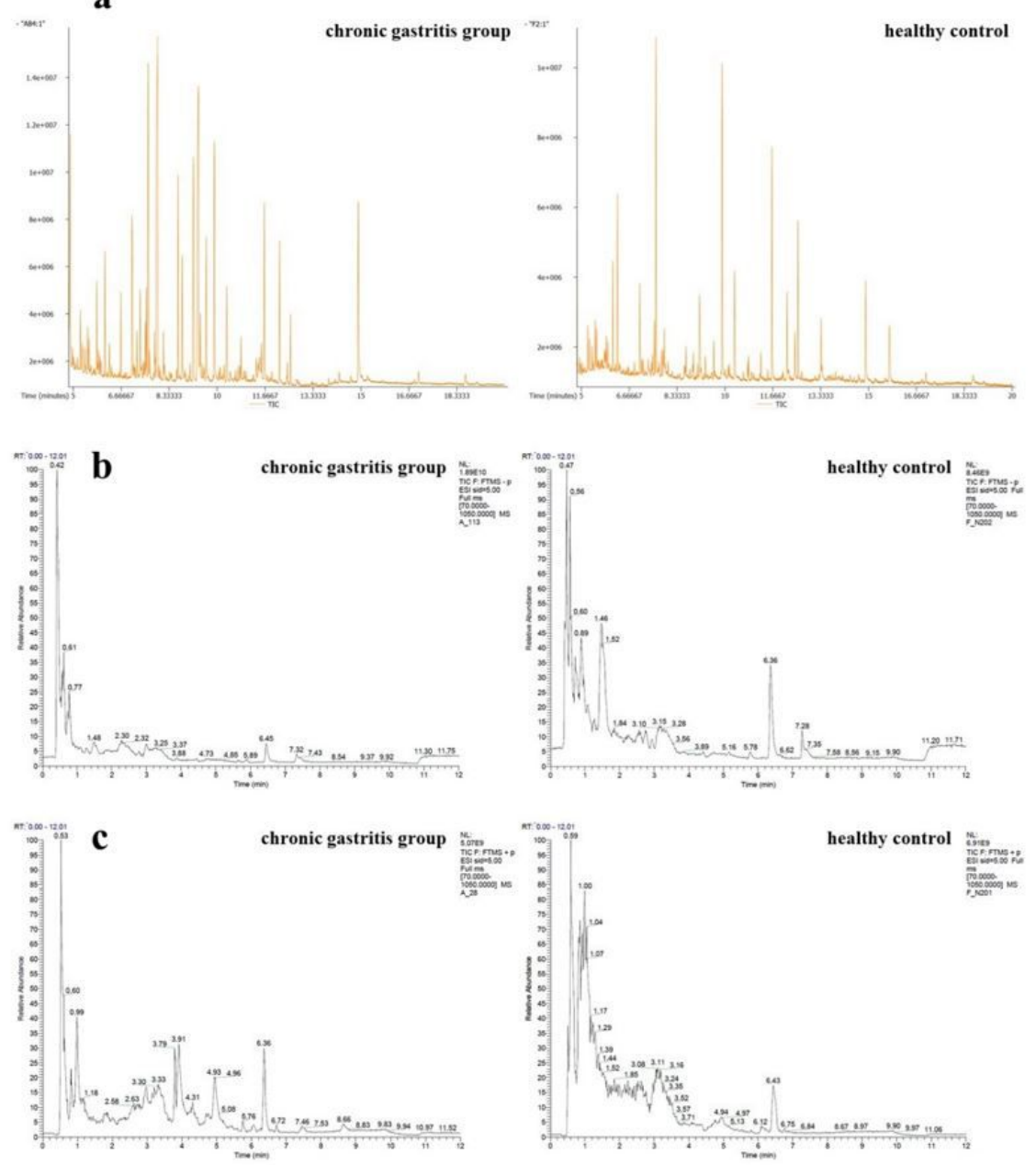

\section{Figure 1}

Mass spectrum peaks of GPL group and healthy control group. a: GC-TOF-MS, b: UHPLC-QE-MS negative ion modes, c: UHPLC-QE-MS positive ion modes. As the examples of the GC-TOF-MS and UHPLC-QE-MS spectra, there were some different mass spectrum peaks between the chronic gastritis patients and healthy control people 


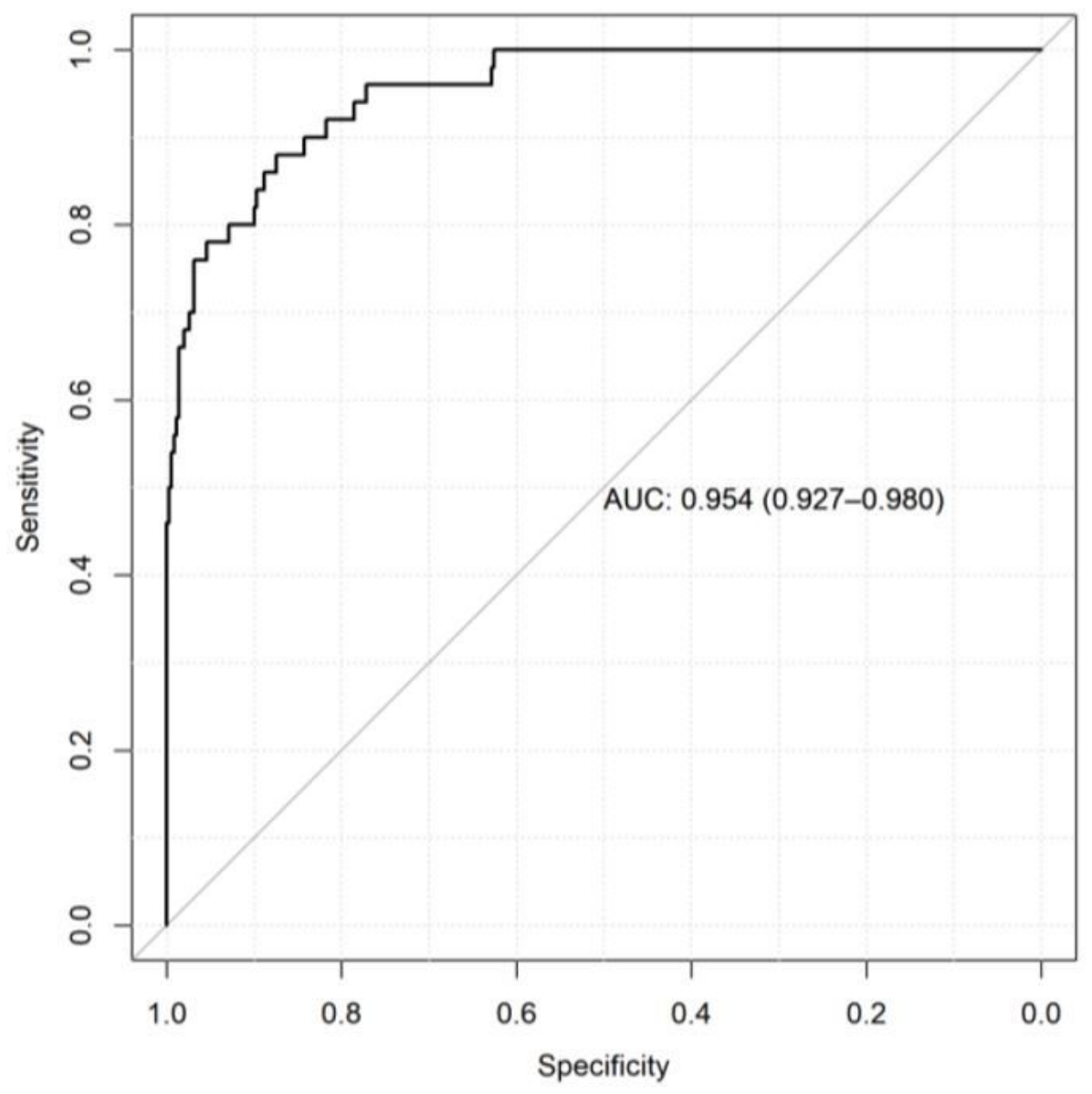

Figure 2

Diagnostic model of tongue coating metabolites in patients with chronic gastritis. The accuracy of the diagnostic model is $95.4 \%$, the specificity is $87.4 \%$, and the sensitivity is $88.0 \%$. 


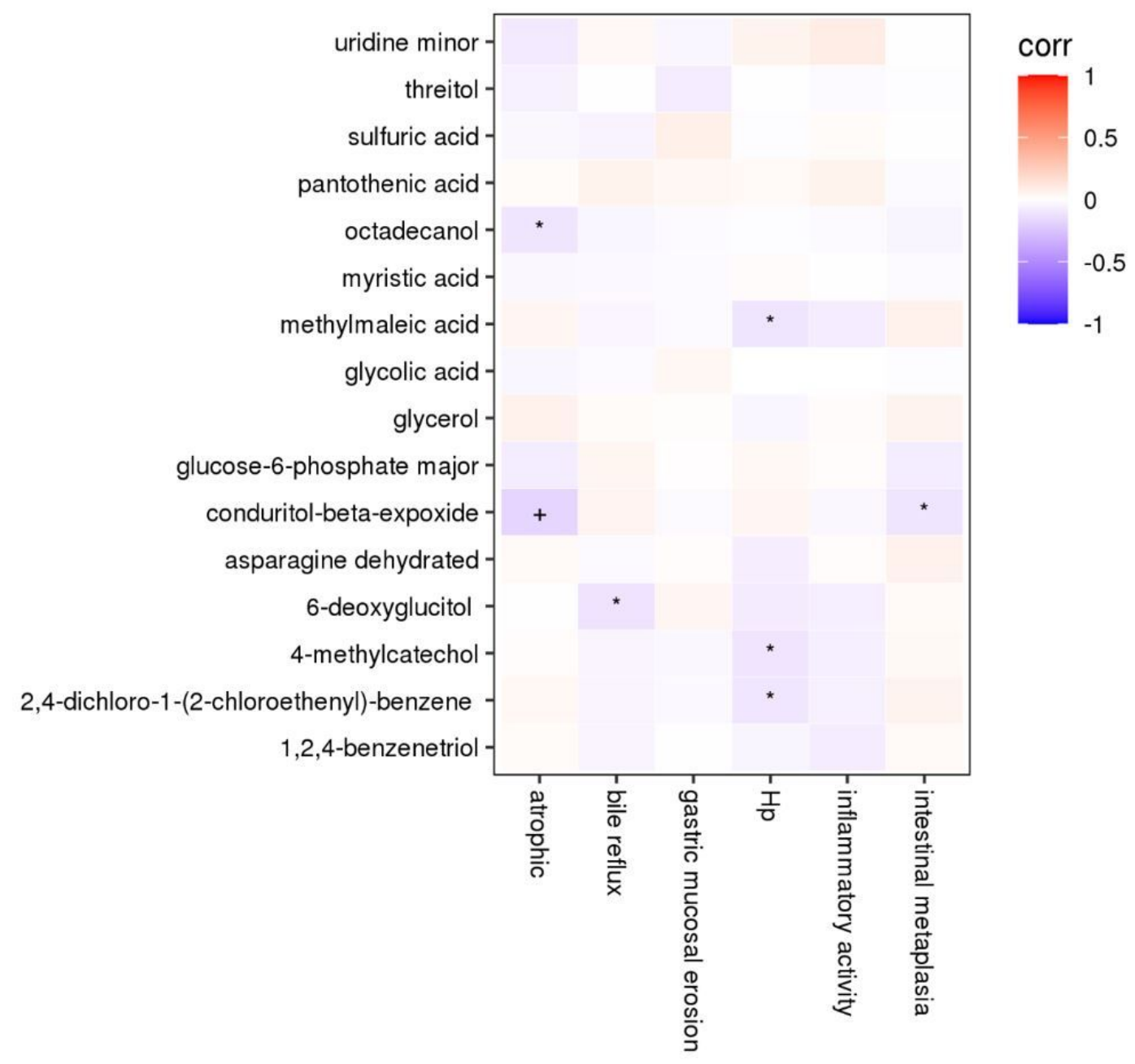

\section{Figure 3}

Figure 3-1. Correlation figure between metabolic markers of tongue coating and gastroscopic indexes and pathological indexes in patients with chronic gastritis analyzed by GC-TOF-MS. Draw a thermodynamic diagram to show the correlation analysis results, which were represented by red (corr $=1$ ), blue $($ corr $=-1)$ and white $($ corr $=0)$. Data with correlation P values less than 0.05 were marked with " " and " + " in the figures. The abscissa was the differential metabolic marker and the ordinate was the gastroscopic characteristic indicator. It can be seen that octadecanol, and conduritol-beta-expoxide were negatively correlated with atrophic; 6-deoxyglucitol was negatively correlated with bile reflux; methylmaleic acid, 4-methylcatechol, and 2,4-dichloro-1-(2-chloroethenyl)-benzene were negatively correlated with $\mathrm{Hp}$; conduritol-beta-expoxide was negatively correlated with intestinal metaplasia. 


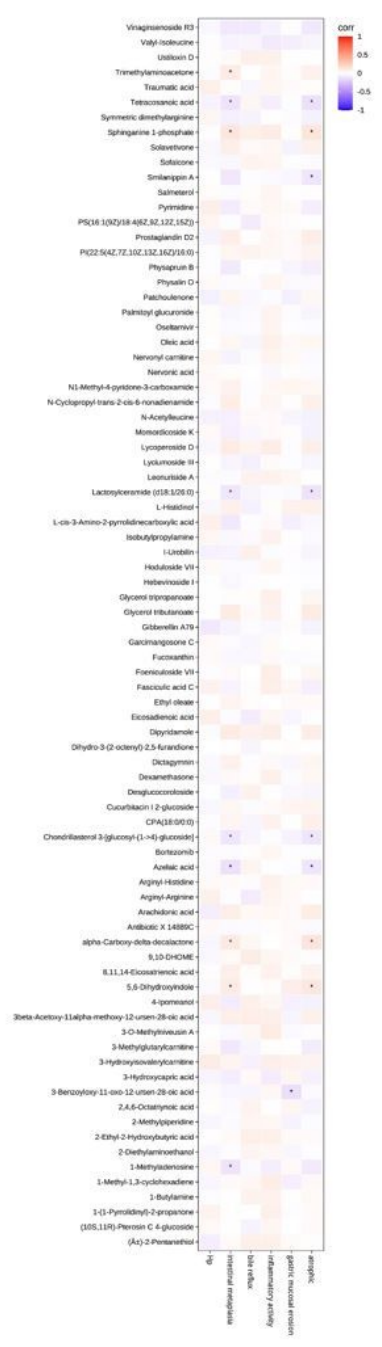

\section{Figure 4}

Figure 3-2. Correlation figure between metabolic markers of tongue coating and gastroscopic indexes and pathological indexes in patients with chronic gastritis analyzed by UHPLC-QE-MS. Draw a thermodynamic diagram to show the correlation analysis results, which were represented by red (corr $=1$ ), blue $($ corr $=-1)$ and white (corr = 0). Data with correlation P values less than 0.05 were marked with " $\star$ " in the figures. The abscissa was the differential metabolic marker and the ordinate was the gastroscopic characteristic indicator. It can be seen that Trimethylaminoacetone, Sphinganine1-phosphate, alpha-Carboxydelta-decalactone, and 5,6-Dihydroxyindole were positively correlated with intestinal metaplasia. Tetracosanoic acid, Lactosylceramide(d18:1/26:0), Chondrillasterol 3-[glucosyl-(1->4)-glucoside], Azelaic acid, and 1-Methyladenosine were negatively correlated with intestinal metaplasia. 3-benzoyloxy-11-oxo12-ursen-28-oic acid was negatively correlated with gastric mucosal erosion. Sphinganine1-phosphate, alpha-Carboxy-delta-decalactone, and 5,6Dihydroxyindole were positively correlated with atrophic. Tetracosanoic acid, Smilanippin A, Lactosylceramide(d18:1/26:0), Chondrillasterol 3-[glucosyl-(1->4)glucoside], and Azelaic acid were negatively correlated with atrophic. 


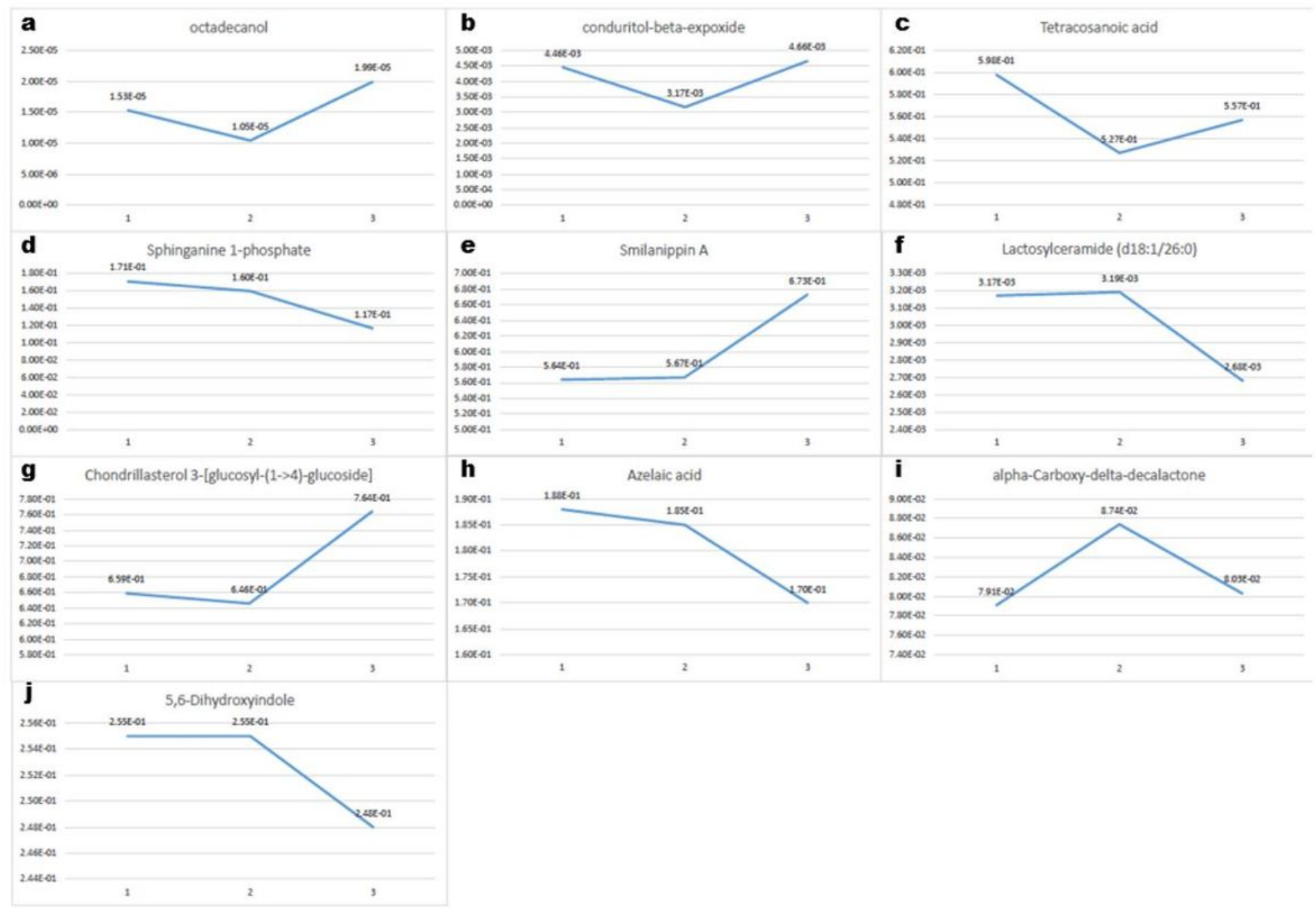

\section{Figure 5}

Figure 4-1. The change figure of tongue coating metabolites in chronic gastritis patients with different degrees of atrophic. The abscissa "1, 2, and 3" represent "light, medium, and severe", and the ordinate represents the content of metabolites. The changing trend of alpha-Carboxy-delta-decalactone content in the tongue coatings of atrophic patients was first increased and then decreased. The changing trend of octadecanol, conduritol-beta-expoxide, Tetracosanoic acid, and Chondrillasterol 3-[glucosyl-(1->4)-glucoside] contents in the tongue coatings of atrophic patients was first decreased and then increased. The content of Smilanippin A in the tongue coating of atrophic patients showed an upward trend. The contents of Sphinganine 1-phosphate, Lactosylceramide (d18:1/26:0), Azelaic acid, and 5,6-Dihydroxyindole in the tongue coating of atrophic patients showed a downward trend. 


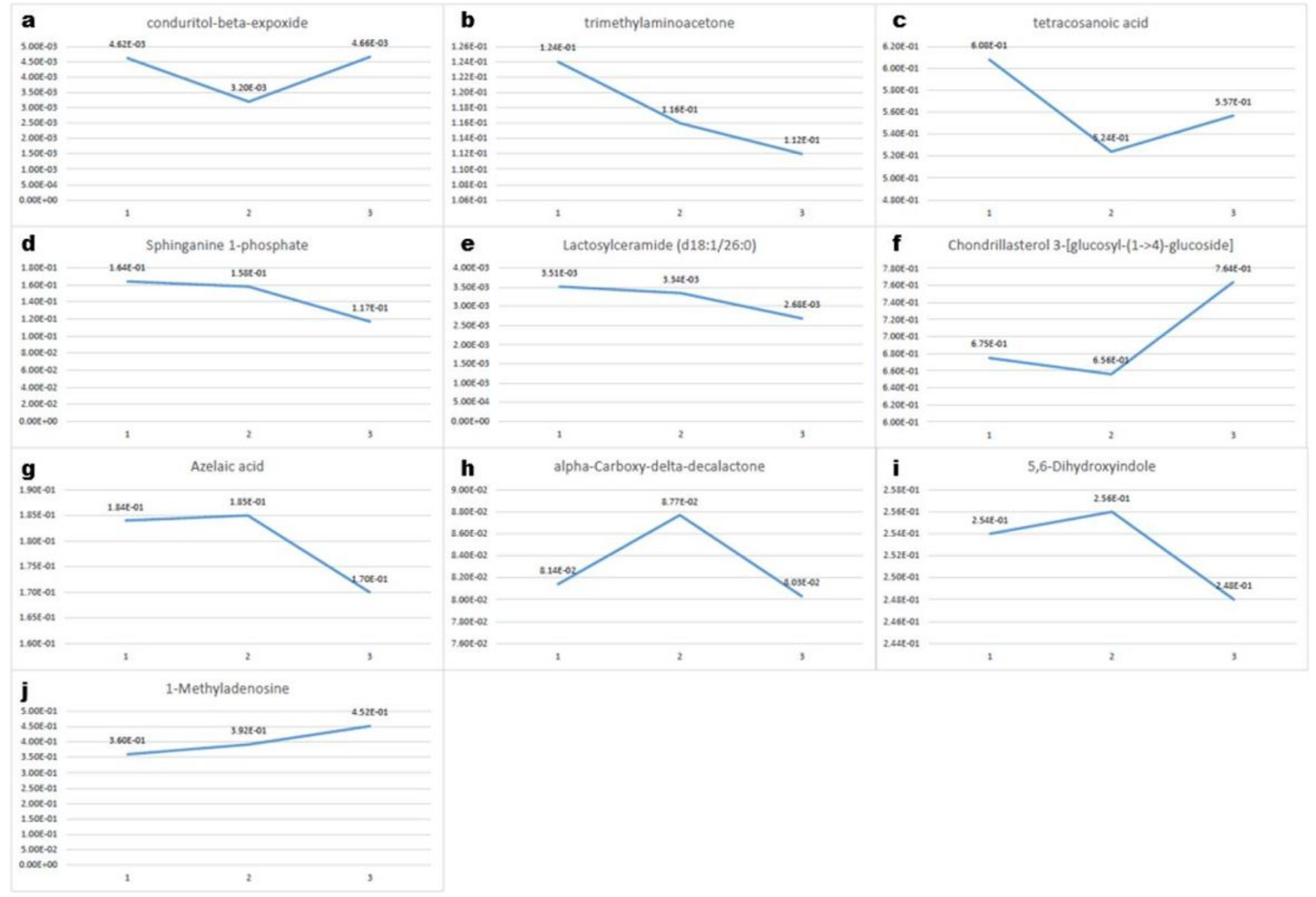

Figure 6

Figure 4-2. The change figure of tongue coating metabolites in chronic gastritis patients with different degrees of intestinal metaplasia. The abscissa "1, 2, and 3" represent "light, medium, and severe", and the ordinate represents the content of metabolites. The changing trend of Azelaic acid, alpha-Carboxy-deltadecalactone, and 5,6-Dihydroxyindole contents in the tongue coatings of intestinal metaplasia patients was first increased and then decreased. The changing trend of conduritol-beta-expoxide, tetracosanoic acid, and Chondrillasterol 3-[glucosyl-(1->4)-glucoside] contents in the tongue coatings of intestinal metaplasia patients was first decreased and then increased. The content of 1-Methyladenosine in the tongue coating of intestinal metaplasia patients showed an upward trend. The contents of trimethylaminoacetone, Sphinganine 1-phosphate, Lactosylceramide (d18:1/26:0) in the tongue coating of intestinal metaplasia patients showed a downward trend.
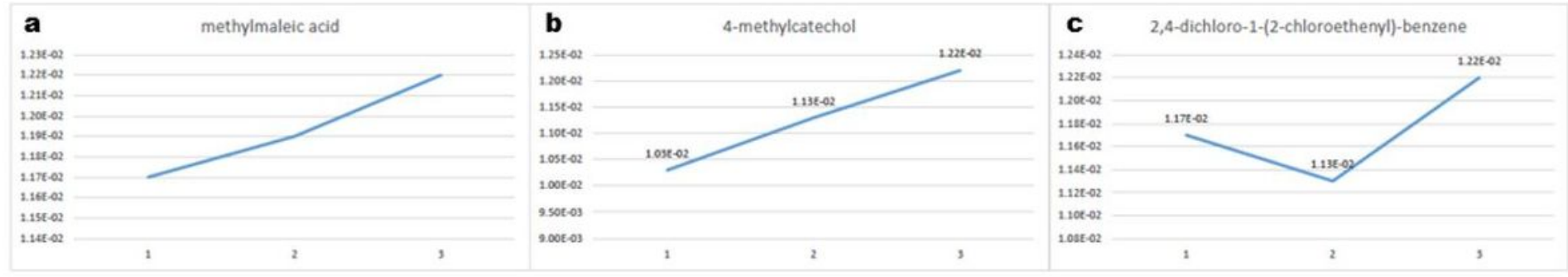

Figure 7

Figure 4-3. The change figure of tongue coating metabolites in chronic gastritis patients with different degrees of Hp infection. The abscissa "1, 2, and 3" represent "light, medium, and severe", and the ordinate represents the content of metabolites. The contents of Methylmaleic acid and 4-methylcatechol in the tongue coating of $\mathrm{Hp}$ infection patients showed an upward trend. The changing trend of 2,4-dichloro-1-(2-chloroethenyl)-benzene content in the tongue coatings of $\mathrm{Hp}$ infection patients was first decreased and then increased.

\section{Supplementary Files}


This is a list of supplementary files associated with this preprint. Click to download.

- tables1.docx

Page 24/24 